ARTICLE

DOI: $10.1038 / \mathrm{s} 41467-018-05043-5$

\title{
The potassium channel KCNJ13 is essential for smooth muscle cytoskeletal organization during mouse tracheal tubulogenesis
}

Wenguang Yin (10 1, Hyun-Taek Kim', ShengPeng Wang2,3, Felix Gunawan (1D 1, Lei Wang2, Keishi Kishimoto (D) ${ }^{4}$, Hua Zhong ${ }^{5}$, Dany Roman ${ }^{5}$, Jens Preussner ${ }^{6}$, Stefan Guenther ${ }^{6}$, Viola Graef ${ }^{1}$, Carmen Buettner ${ }^{1}$, Beate Grohmann ${ }^{1}$, Mario Looso ${ }^{6}$, Mitsuru Morimoto (D) ${ }^{4}$, Graeme Mardon ${ }^{5}$, Stefan Offermanns ${ }^{2,7}$ \& Didier Y.R. Stainier ${ }^{1}$

Tubulogenesis is essential for the formation and function of internal organs. One such organ is the trachea, which allows gas exchange between the external environment and the lungs. However, the cellular and molecular mechanisms underlying tracheal tube development remain poorly understood. Here, we show that the potassium channel KCNJ13 is a critical modulator of tracheal tubulogenesis. We identify Kcnj13 in an ethylnitrosourea forward genetic screen for regulators of mouse respiratory organ development. Kcnj13 mutants exhibit a shorter trachea as well as defective smooth muscle (SM) cell alignment and polarity. KCNJ13 is essential to maintain ion homeostasis in tracheal SM cells, which is required for actin polymerization. This process appears to be mediated, at least in part, through activation of the actin regulator AKT, as pharmacological increase of AKT phosphorylation ameliorates the Kcnj13-mutant trachea phenotypes. These results provide insight into the role of ion homeostasis in cytoskeletal organization during tubulogenesis.

\footnotetext{
${ }^{1}$ Department of Developmental Genetics, Max Planck Institute for Heart and Lung Research, Bad Nauheim 61231, Germany. ${ }^{2}$ Department of Pharmacology, Max Planck Institute for Heart and Lung Research, Bad Nauheim 61231, Germany. ${ }^{3}$ Cardiovascular Research Center, School of Basic Medical Sciences, Xi'an Jiaotong University, Xi'an 710061, China. ${ }^{4}$ Laboratory for Lung Development, RIKEN Center for Developmental Biology, Kobe 650-0047, Japan. ${ }^{5}$ Departments of Pathology and Immunology and Molecular and Human Genetics, Integrative Molecular and Biomedical Sciences Program, Baylor College of Medicine, Houston, TX 77030, USA. ${ }^{6}$ Max Planck Institute for Heart and Lung Research, ECCPS Bioinformatics and Deep Sequencing Platform, Bad Nauheim 61231, Germany. ${ }^{7}$ Center for Molecular Medicine, Goethe University, Frankfurt 60590, Germany. Correspondence and requests for materials should be addressed to W.Y. (email: Wenguang.Yin@mpi-bn.mpg.de) or to D.Y.R.S. (email: Didier.Stainier@mpi-bn.mpg.de)
} 
T he trachea consists of endoderm-derived epithelium surrounded by mesoderm-derived cartilage, connective tissue, and smooth muscle $(\mathrm{SM})^{1}$. The $\mathrm{SM}$ provides the elasticity necessary to control tracheal contraction, whereas the cartilage provides tissue rigidity to prevent airway collapse ${ }^{2}$. In humans, tracheal tube formation defects have been reported to cause tracheomalacia, which is characterized by deficiency of the supporting cartilage and may lead to airway collapse, respiratory distress, and death ${ }^{3}$. Studies on the cellular and molecular mechanisms underlying tracheal tubulogenesis have mostly focused on the role of epithelial cells ${ }^{4,5}$ as well as the complex signaling between the epithelium and mesenchyme $e^{6,7}$, but how SM cells regulate this process remains unknown.

Another poorly studied aspect of tubulogenesis is the potential role of ion channels and their mediated ion homeostasis. Recent data indicate that potassium channels have important roles in the behavior of non-excitable cells, including in tissue patterning ${ }^{8,9}$. In this context, cytoskeletal organization, including the modulation of actin dynamics, is essential to maintain cell shape and alignment. Amongst a variety of actin-associated factors, the serine/threonine kinase $\mathrm{AKT}$ has emerged as one important regulator of actin organization ${ }^{10,11}$. AKT phosphorylates Girdin, an actin-binding protein that regulates F-actin levels, and phosphorylated Girdin accumulates at the leading edge of migrating cells $^{11}$. These data provide a direct link between AKT activity and actin polymerization. However, how AKT activity is regulated is poorly understood.

Here, starting with a forward genetic approach in mouse, we reveal a role for a specific potassium channel, and ion homeostasis, during tracheal tubulogenesis, at least in part via the control of actin dynamics and cytoskeletal organization.

\section{Results}

Kcnj13 $3^{T 38 C / T 38 C}$ mice exhibit tracheal defects. We sought to identify regulators of mouse respiratory organ formation by conducting a large-scale forward genetic screen using ethylnitrosourea (ENU) mutagenesis. Mutagenized C57BL/6J mice were bred to uncover recessive phenotypes using a two generation backcross breeding scheme (Supplementary Fig. 1a). Early postnatal tracheas were examined using alcian blue to label cartilage, and alpha SM actin (aSMA) immunostaining in whole-mount preparations (Fig. 1a). We screened 473 G1 animals and recovered five mutants with tracheal tube formation defects, one of which displayed cyanosis (Fig. 1b) and neonatal respiratory distress (Fig. 1c and Supplementary Movie 1), and died within $24 \mathrm{~h}$ of birth. These mutant animals were born in the expected Mendelian ratio with a shortened (Fig. 1d, e) and collapsed (Fig. 1f, g) trachea, and fractured cartilage rings instead of the intact ventrolateral cartilage rings seen in wild-type (WT) siblings (Fig. 1d, f). In addition, disorganized SM, characterized by the narrowing of SM stripes, was observed in the mutants (Fig. 1h). To examine the lungs, we performed hematoxylin and eosin staining of tissue sections. Mutants exhibited reduced airspace and thickened walls in distal regions compared to WT (Fig. 1i, j). These data indicate that the mutants die from compromised respiratory function due to defective formation of the tracheal tube and respiratory air sacs.

To identify the phenotype-causing mutation, we conducted whole-exome sequencing of G4 genomic DNA samples (Supplementary Fig. 1b), and identified Kcnj13, which encodes a member of the inwardly rectifying potassium channel family, as a candidate gene (Fig. 1k). The identified allele carries a mutation that causes a leucine-to-proline substitution at the highly conserved $13^{\text {th }}$ residue (c.38T $>C$ (p.Leu13Pro)). Next, we carried out genetic linkage analysis by genotyping 105 G4- and
G5-mutant animals, and found complete linkage between the tracheal phenotype and the Kcnj13 ${ }^{T 38 C / T 38 C}$ allele (Fig. 11). We then performed a complementation test by crossing mice carrying the ENU-induced Kcnj13 allele $\left(K c n j 13^{T 38 C /+}\right.$ ) with mice carrying a Kcnj13 deletion allele (Supplementary Fig. 2a-d), and found that complementation did not occur in the Kcnj13 ${ }^{\text {Del-E2 } \& E 3 / T 38 C}$ double heterozygous animals (Fig. $1 \mathrm{~m}$ ), indicating that loss of $\mathrm{KCNJ} 13$ function is likely responsible for the observed tracheal phenotypes. To further test the role of Kcnj13 in tracheal development, we analyzed tracheal formation

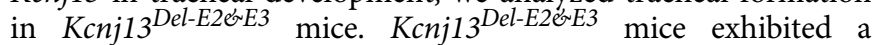
shortened trachea with fractured cartilage rings (Supplementary Fig. 3a, b) and disorganized SM (Supplementary Fig. 3c, d), and died within $24 \mathrm{~h}$ of birth, similar to Kcnj13 ${ }^{\text {T38C/T38C }}$ animals. We then examined the spatial and temporal expression pattern of Kcnj13 in the developing mouse trachea and lungs. Kcnj13 mRNA was expressed at low levels in E11.5-E13.5 tracheas (Supplementary Fig. 4a and Supplementary Table 1). At E13.5 and E14.5, KCNJ13 protein was clearly detected in tracheal SM (Fig. 2a), but not in $\mathrm{SOX}^{+}$mesenchymal cells (Fig. 2b). After E15.5, KCNJ13 was also detected in a subset of tracheal epithelial cells (Fig. 2c). In the lungs, Kcnj13 mRNA was detected in epithelial cells as early as E16.5 (Supplementary Fig. 4b). KCNJ13 was expressed in epithelial cells of bronchioles (Fig. 2d, e) and alveolar type II cells (Fig. 2f), similar to the expression of a Kcnj13 BAC reporter ${ }^{12}$. Interestingly, KCNJ13 protein was still detectable at WT levels in Kcnj13 ${ }^{\mathrm{T} 38 \mathrm{C} / \mathrm{T} 38 \mathrm{C}}$ tracheas (Supplementary Fig. 4c), indicating that the c.38T $>\mathrm{C}$ mutation has no effect on protein stability. Altogether, these results indicate that loss of Kcnj13 function causes severe tracheal defects.

$K c n j 13^{T 38 C / T 38 C}$ mice exhibit defects in tracheal elongation. To examine the formation of the trachea in detail, we performed a systematic analysis of tracheal tube development.

Tracheal tube length did not significantly differ between Kcnj13 ${ }^{T 38 C / T 38 C}$ mice and their WT siblings from E11.5 to E13.5 (Fig. 3a, b). However, starting at E14.5, we observed that Kcnj13 ${ }^{\text {T38C/T38C }}$ tracheas were shorter than WT (Fig. 3a, b), indicating that impaired tracheal tube elongation occurs after SM differentiation, which starts at E11.52. Since altered SM morphogenesis can affect tracheal elongation ${ }^{13}$, we analyzed tracheal SM development. SM cells which are positioned dorsally in the trachea, displayed no obvious differences between WT and Kcnj13 ${ }^{T 38 C / T 38 C}$ animals from E11.5 to E12.5 (Fig. 3c, d). Disorganized SM stripes of decreased area were first observed in E13.5 Kcnj13 ${ }^{\text {T38C/T38C }}$ tracheas and became more noticeable starting at E14.5 (Fig. 3c, d). Another important process during trachea formation is the appearance of cartilage from the condensation of mesenchymal cells into chondrogenic nodules ${ }^{6}$. At E13.5, a clear pattern of condensed SOX9 ${ }^{+}$mesenchymal cells resembling cartilaginous rings was readily distinguished in $\mathrm{WT}$, whereas such condensations were seldom detected in Kcnj13 ${ }^{\text {T38C }}$ T38C tracheas (Fig. 3e, f). This effect on SM organization and mesenchymal condensation appeared specific, as we did not observe significant differences between WT and mutant animals in the proliferation of SM cells (Supplementary Fig. 5a, b) or SOX9 ${ }^{+}$mesenchymal cells (Supplementary Fig. 5c, d) or apoptosis (Supplementary Fig. 5e). Next, we examined the expression levels of Sox9, which encodes a key regulator of chondrogenic nodule formation ${ }^{14}$. Kcnj13 $3^{\text {T38C/T38C }}$ tracheas exhibited no significant difference in Sox9 mRNA levels compared to WT (Supplementary Fig. 5f and Supplementary Table 2), indicating that Kcnj13 participates in cartilage formation through a Sox9 independent pathway. Potassium channels have 
a
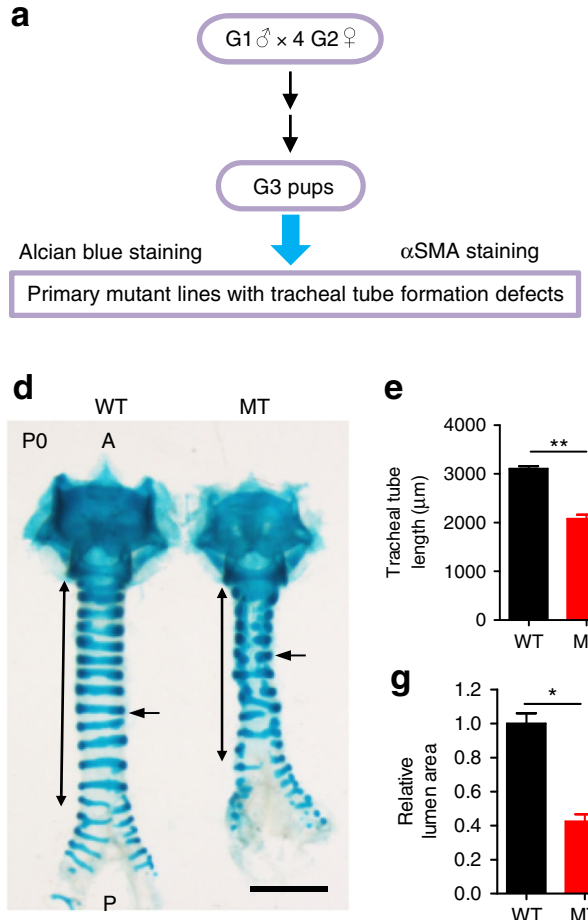

e
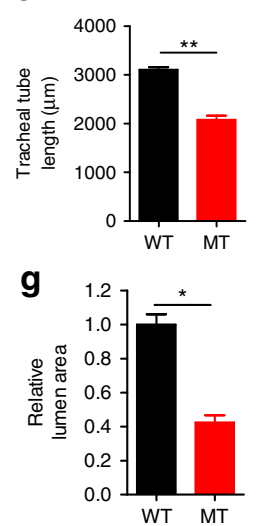

b

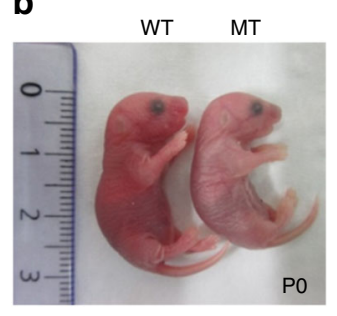

C

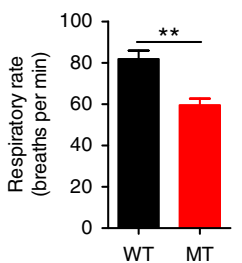

h
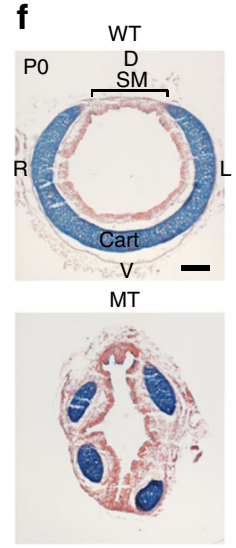

j

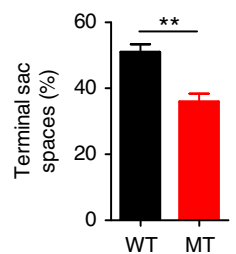

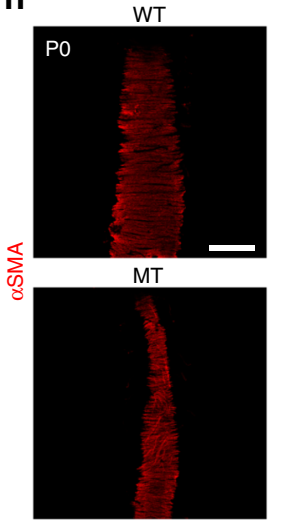

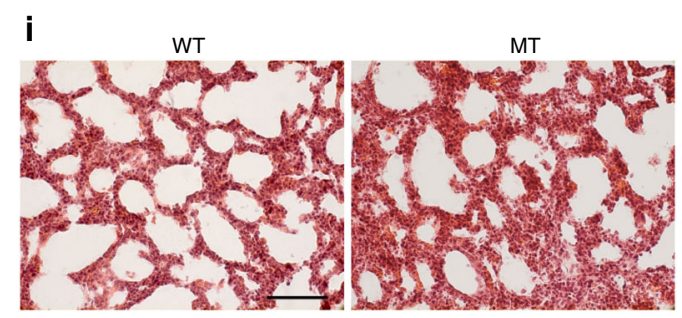

k

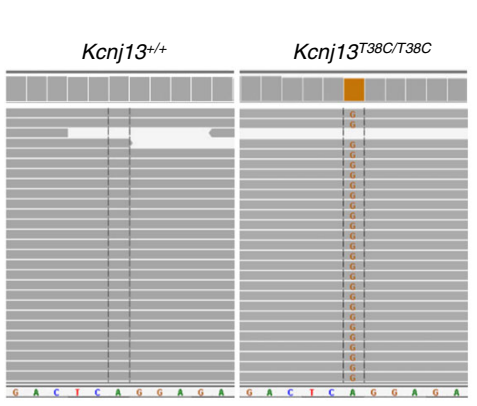

I

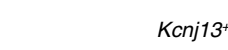

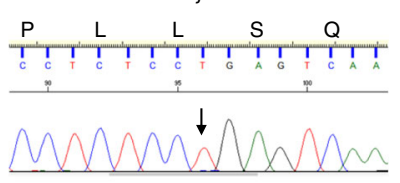

Kcnj13 $3^{\text {T38C/T38C }}$

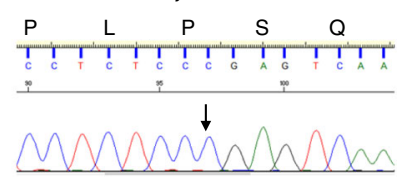

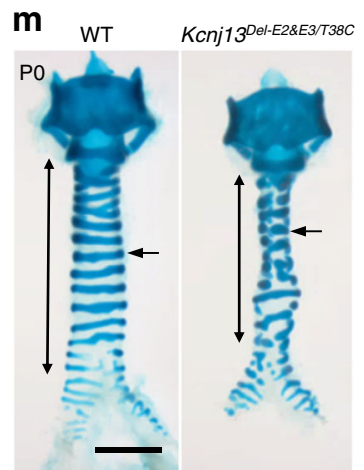

Fig. $1 \mathrm{Kcnj73} 3^{T 38 C / T 38 C}$ mice exhibit tracheal tube formation defects. a Workflow for ENU screen in mouse to identify mutants with respiratory organ formation defects. b Representative gross morphology of PO WT $(n=12)$ and mutants $(n=12)$. c Quantification of PO WT $(n=12)$ and mutant $(n=12)$ respiratory rates. $\mathbf{d}$ Representative image of ventral view of whole-mount tracheas stained with alcian blue from PO WT $(n=35)$ and mutants $(n=35)$. Double-sided arrows indicate tracheal tube length. Arrows point to tracheal cartilage rings. e Quantification of PO WT (10) and mutant (10) tracheal tube length. $\mathbf{f}$ Representative images of transverse sections of tracheas stained with alcian blue and nuclear fast red from PO WT $(n=9)$ and mutants $(n=9)$. $\mathbf{g}$ Quantification of PO WT $(n=9)$ and mutant (9) tracheal lumen area. $\mathbf{h}$ Representative images of dorsal views of whole-mount tracheas stained for $\alpha$ SMA (red) from PO WT $(n=10)$ and mutants $(n=10)$. i Representative images of lung tissue sections stained with hematoxylin and eosin from PO WT $(n=8)$ and mutants $(n=8)$. $\mathbf{j}$ Quantification of PO WT $(n=8)$ and mutant (8) terminal airspaces in the lungs. $\mathbf{k}$ Whole-exome sequencing of PO WT ( $n=$ 2 ) and mutant $(n=2)$ genomic DNA. Green indicates the WT nucleotide A. Light brown indicates the mutant nucleotide G. I Sequence of WT and mutant genomic DNA around the lesion. DNA sequence fluorogram shows CTG for leucine in WT ( $n=119)$ (upper panel) and CCG for proline in mutants ( $n=$ 105) (lower panel). Arrows point to the mutation site. $\mathbf{m}$ Representative images of ventral views of whole-mount tracheas stained with alcian blue from PO WT $(n=17)$ and Kcnj13 Del-E2\&E3/T38C double heterozygous animals $(n=17)$. Double-sided arrows indicate tracheal tube length. Arrows point to tracheal cartilage rings. Scale bars: $1000 \mu \mathrm{m}(\mathbf{d}, \mathbf{m}), 500 \mu \mathrm{m}(\mathbf{h}), 100 \mu \mathrm{m}(\mathbf{e}, \mathbf{i}) .{ }^{\star} P<0.05$; ${ }^{\star} P<0.01$; unpaired Student's $t$-test, mean \pm s.d. WT: wild-type, MT: mutant, A: anterior, P: posterior, D: dorsal, V: ventral, L: left, R: right, Cart: cartilage, SM: smooth muscle 
a

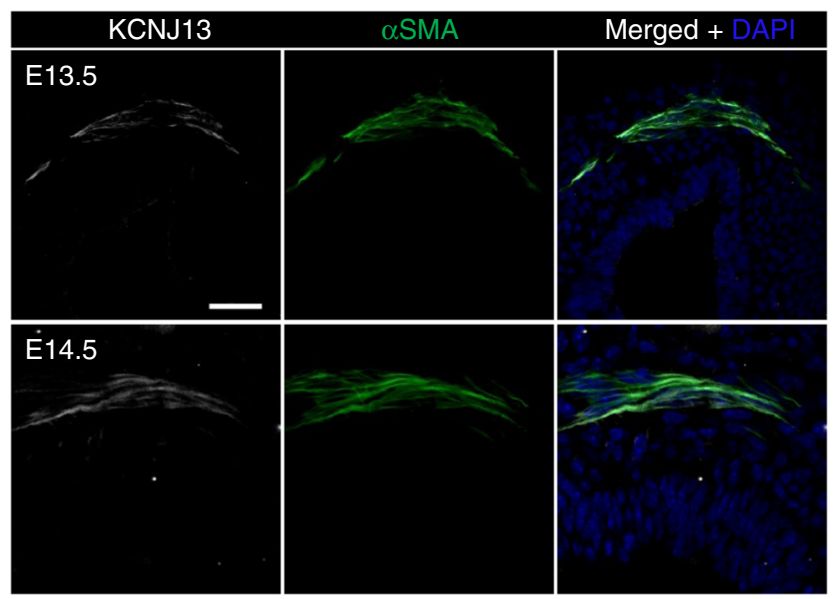

C

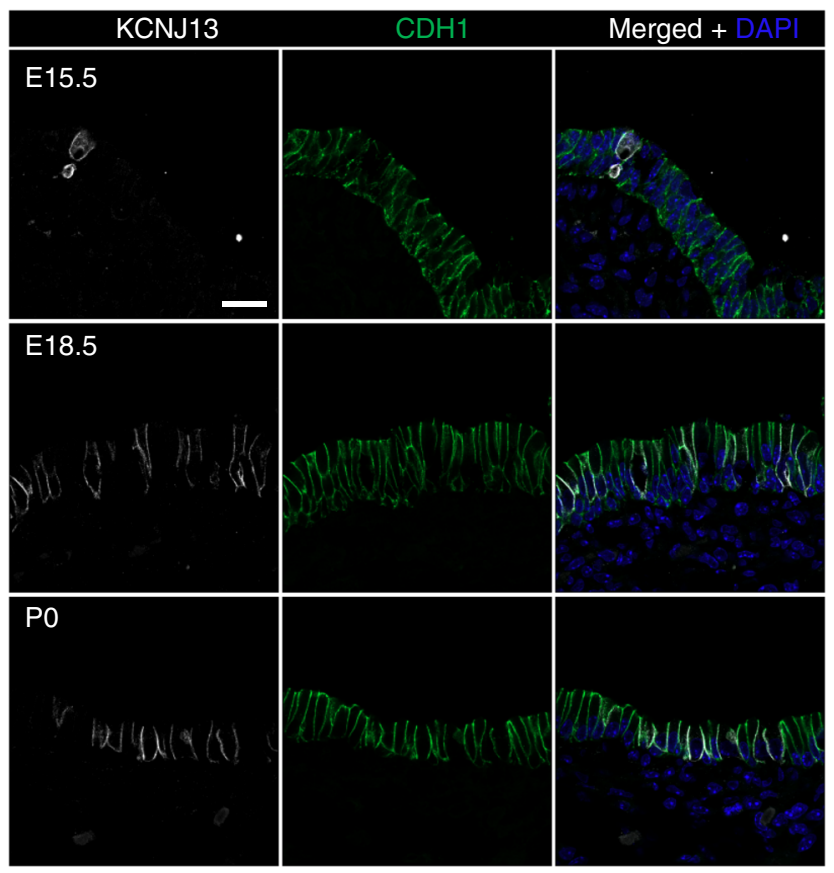

b

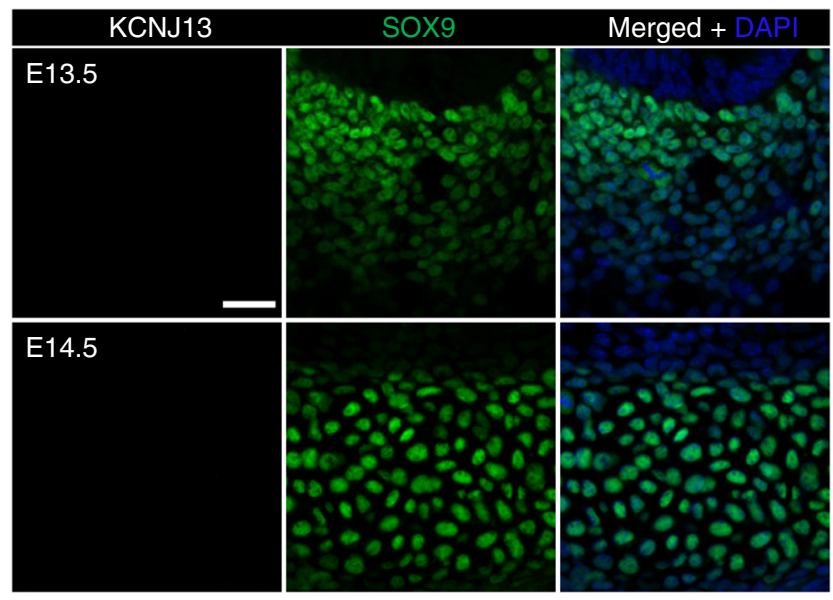

d

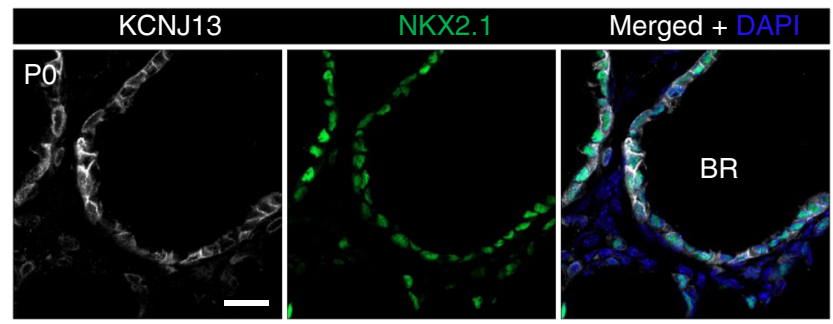

e

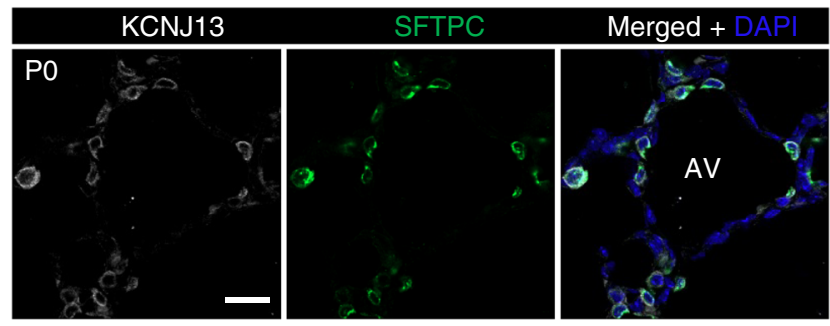

Fig. 2 Kcnj13 is expressed in SM and epithelial cells in the trachea, as well as in epithelial cells in the lungs. a Immunostaining for KCNJ13 (red), $\alpha$ SMA (green), and DAPI staining (blue) of transverse sections of E13.5 $(n=8)$ and E14.5 $(n=8)$ WT tracheas. b Immunostaining for KCNJ13 (red), SOX9 (green), and DAPI staining (blue) of transverse sections of E13.5 $(n=8)$ and E14.5 $(n=8)$ WT tracheas. c Immunostaining for KCNJ13 (red), CDH1 (green), and DAPI staining (blue) of transverse sections of E15.5 $(n=8)$, E18.5 $(n=8)$, and PO $(n=8)$ WT tracheas. d Immunostaining for KCNJ13 (red), NKX2.1 (green), and DAPI staining (blue) of PO WT lung tissue sections $(n=7)$. e Immunostaining for KCNJ13 (red), SFTPC (green), and DAPI staining (blue) of PO WT lung tissue sections $(n=7)$. Scale bars: $20 \mu \mathrm{m}$. BR: bronchioles, AV: alveoli

been reported to modulate cell differentiation ${ }^{15}$. We observed reduced differentiation of acetylated alpha-tubulin ${ }^{+}$multiciliated cells (Supplementary Fig. 5g, h) but a WT-like distribution of $\mathrm{CC}{ }^{+}$club cells (Supplementary Fig. $5 \mathrm{~g}, \mathrm{i}$ ) and $\mathrm{KRT}^{+}$basal cells (Supplementary Fig. 5g, j) in the mutant tracheas. In addition, Kcnj13 $3^{T 38 C / T 38 C}$ tracheas exhibited WT-like organization of the tracheal epithelium (Supplementary Fig. 5k). Collectively, these results suggest that SM organization and mesenchymal condensation are essential for tracheal tube formation.

Kcnj13 modulates SM cell alignment and polarity. To investigate the cellular mechanisms underlying Kcnj13-mediated tracheal tube formation, we analyzed SM cell alignment and polarity in WT and Kcnj13 ${ }^{\text {T38C/T38C }}$ tracheas. Tracheal SM cells differentiate and acquire radial cell polarity starting at E11.5 ${ }^{13}$; they develop spindle shapes and become circumferentially aligned by E13.5 (Fig. 3c). WT tracheal SM cells were aligned in a direction perpendicular to that of tracheal elongation by E14.5 (Fig. 4a, b). In contrast, Kcnj13 ${ }^{T 38 C / T 38 C} \mathrm{SM}$ cells displayed random alignment (Fig. 4a), which could be quantitatively assessed (Fig. 4b), as well as abnormally rounded nuclei (Fig. 4a, c). These mutant SM cells aligned into 7-8 layers compared to 3-4 layers in WT at E14.5 (Fig. 4d, e). To better understand the polarization of SM cells, we examined the localization of the Golgi apparatus relative to the cell nucleus, using the cis-Golgi matrix marker GM130, a widely used method to determine cell polarity in different cell types ${ }^{16-18}$. In WT SM cells, the GM130-labeled Golgi exhibited a ribbon-like morphology and localized preferentially by the long 

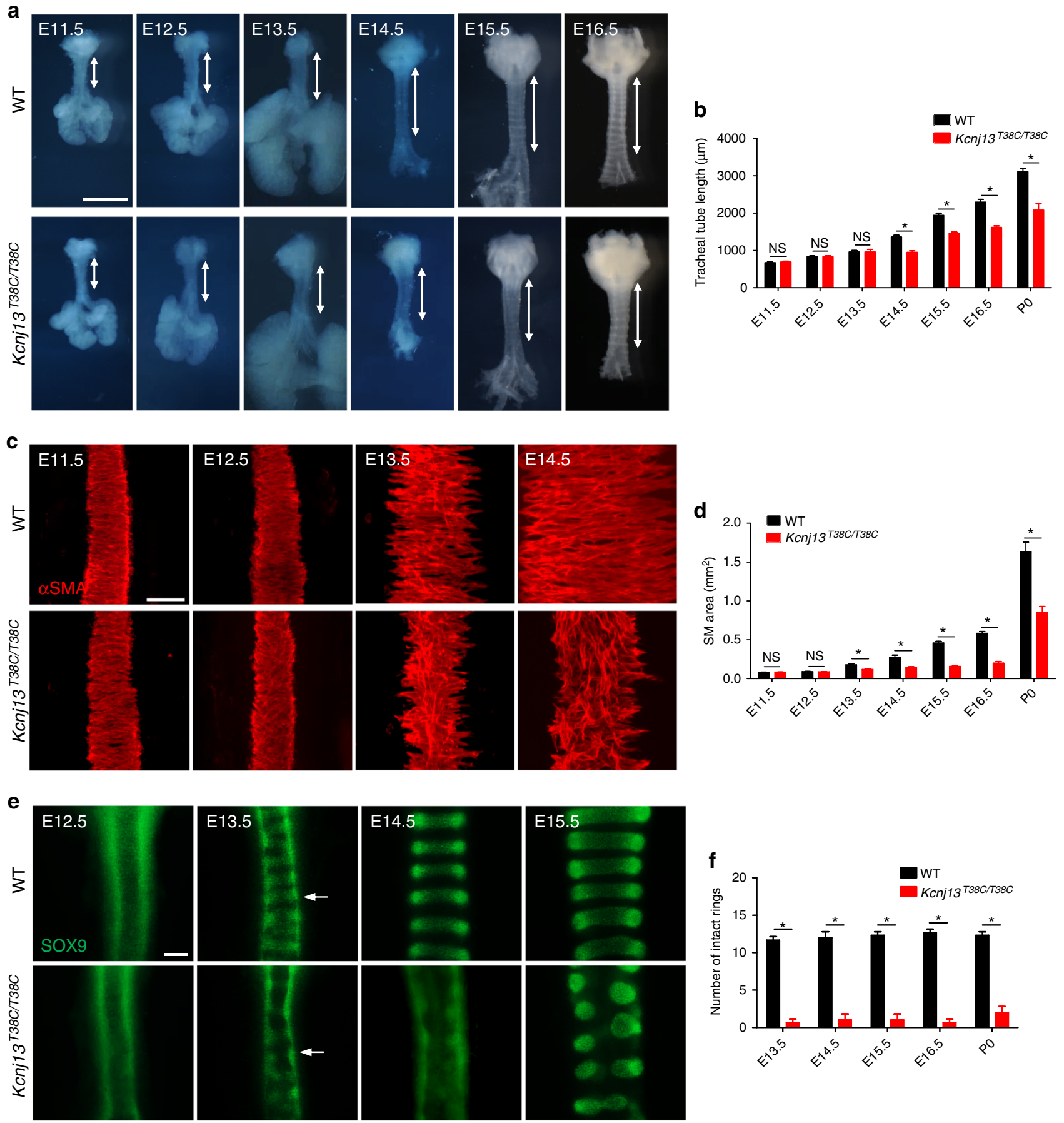

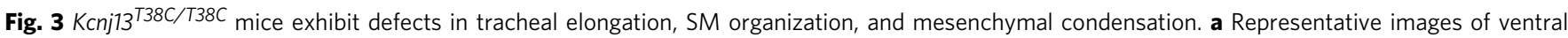
views of WT $(n=8)$ and $K c n j 13^{T 38 C / T 38 C}(n=8)$ tracheas at several embryonic stages. Double-sided arrows indicate tracheal tube length. b Quantification

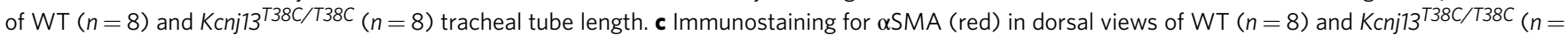
8) tracheas at several embryonic stages. d Quantification of WT $(n=8)$ and Kcnj13 T38C/T38C $(n=8)$ SM area. e Immunostaining for SOX9 (green) in ventral views of WT $(n=8)$ and $K c n j 73^{T 38 C / T 38 C}(n=8)$ tracheas at several embryonic stages. Arrows point to tracheal mesenchymal condensations. f Quantification of intact tracheal mesenchymal condensations in WT $(n=8)$ and $K c n j 13^{T 38 C / T 38 C}(n=8)$ animals. Scale bars: $1000 \mu \mathrm{m}(\mathbf{a}), 100 \mu \mathrm{m}$

(c, e). ${ }^{\star} P<0.05$; NS not significant. Unpaired Student's $t$-test, mean \pm s.d

edges of the nucleus (Fig. 4f, g). In contrast, in $\mathrm{Kcnj13} 3^{\mathrm{T} 38 \mathrm{C} / \mathrm{T} 38 \mathrm{C}}$ SM cells, the Golgi exhibited a more compact structure with random alignment (Fig. 4f, g). Since Wnt5a-Ror2 signaling modulates tracheal SM cell polarity ${ }^{13}$, we examined for changes in Wnt/planar cell polarity (PCP), a pathway known to control cell polarity ${ }^{19}$. Kcnj13 $3^{\text {T38C/T38C }}$ tracheas exhibited no significant differences in expression levels of Wnt/PCP genes
(Supplementary Fig. 6 and Supplementary Table 3), indicating that Kcnj13 signals via a Wnt/PCP independent pathway to direct $\mathrm{SM}$ cell polarity. In addition, $\mathrm{SOX}^{+}$mesenchymal cells displayed a WT-like morphology in Kcnj13 ${ }^{\text {T38C/T38C }}$ tracheas (Supplementary Fig. 7a, b). These findings indicate that KCNJ13 is required to coordinate SM cell polarity and generate tracheal tissue architecture. 
To investigate whether defects in condensation of SOX9 ${ }^{+}$ mesenchymal cells might affect tracheal tube elongation, we deleted Sonic hedgehog $(S h h)$ function in the tracheal epithelium

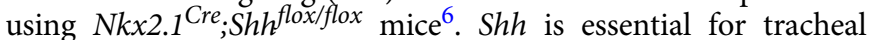
cartilage formation ${ }^{20}$ and epithelial deletion of Shh using Nkx2.1 ${ }^{C r e}$;Shh flox/flox mice leads to mesenchymal cell

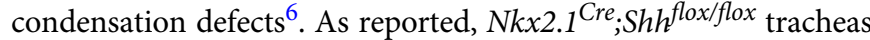
displayed severe $\mathrm{SOX}^{+}$mesenchymal cell condensation defects compared to controls at E16.5 (Supplementary Fig. 8a, b), as observed in Kcnj13 ${ }^{T 38 C / T 38 C}$ tracheas. However, SM cell alignment (Supplementary Fig. 8c, d) or tracheal tube length (Supplementary Fig. 8e, f) did not appear to be affected in these a

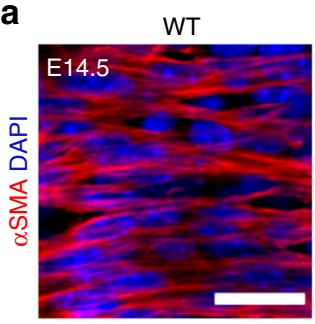

d

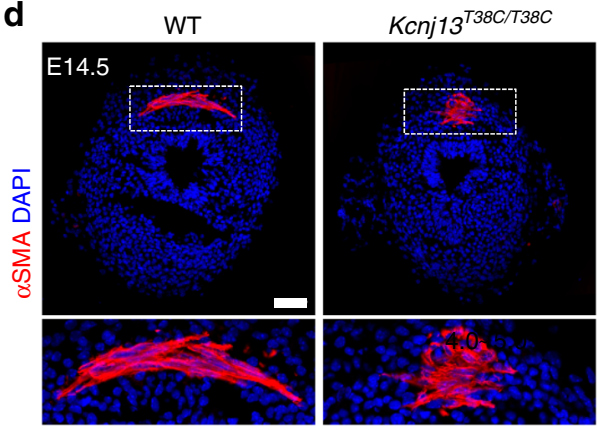

h Tamoxifen Analysis

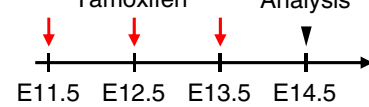

i

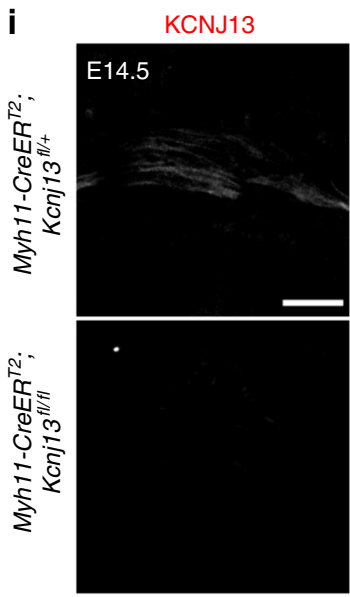

I

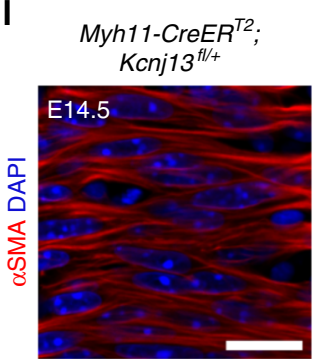

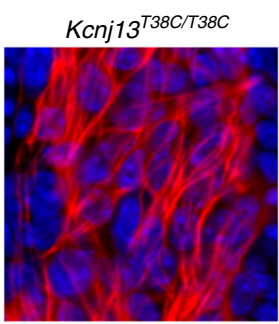
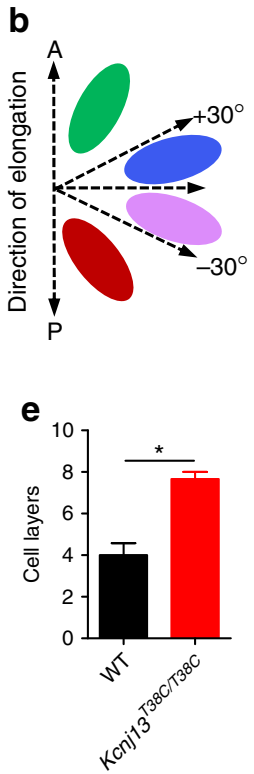

$\alpha$ SMA

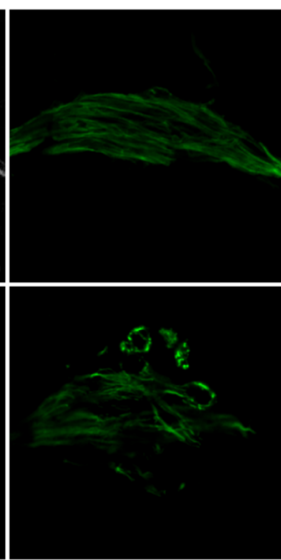

Myh11-CreER ${ }^{T 2}$. Kcnj13 $3^{t / f t}$

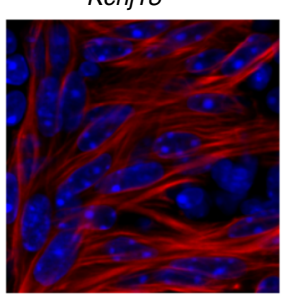

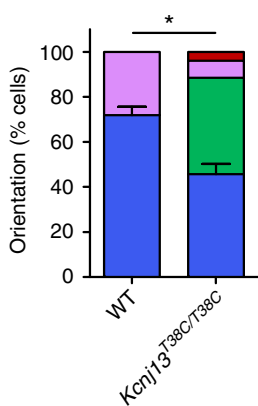

$\mathbf{f}$
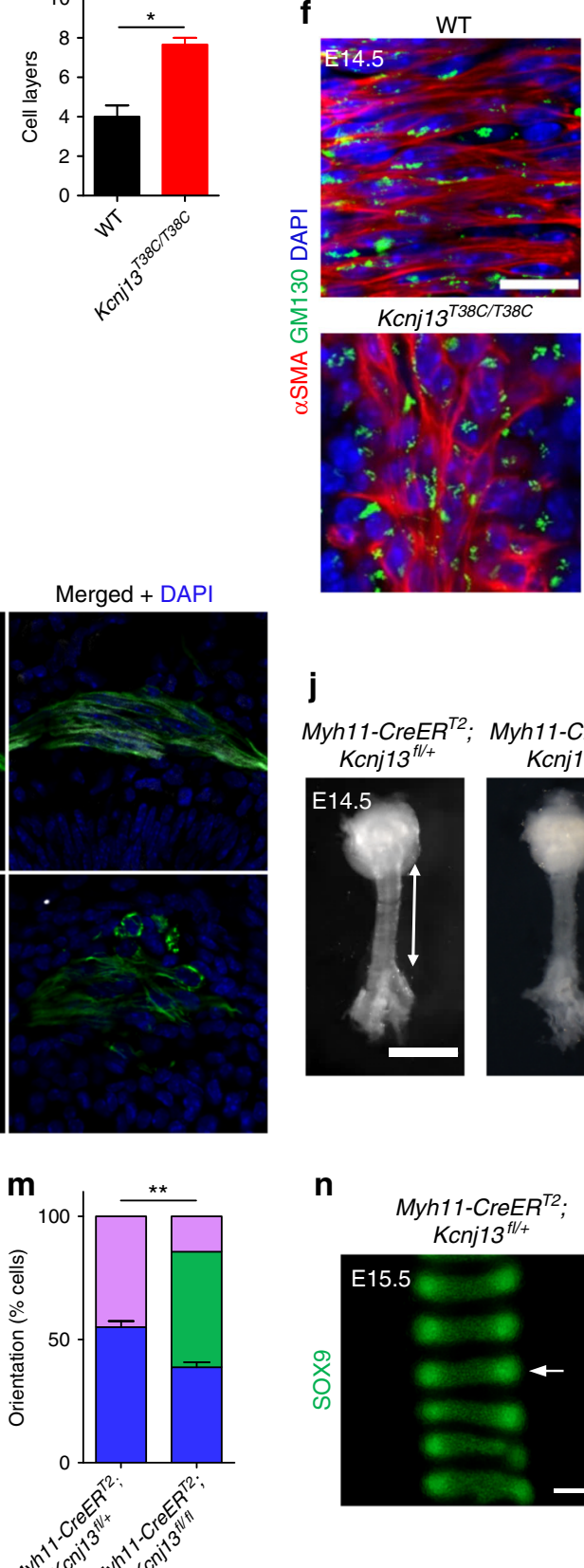

j

Myh11-CreER ${ }^{T 2} ;$ Myh11-CreER ${ }^{T 2}$;
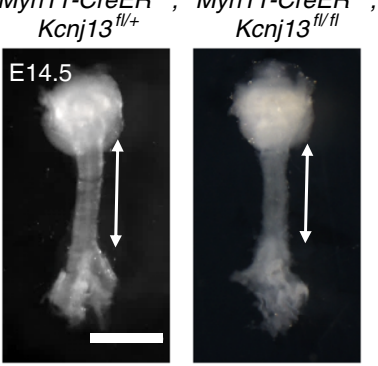

$\mathbf{n}$

C
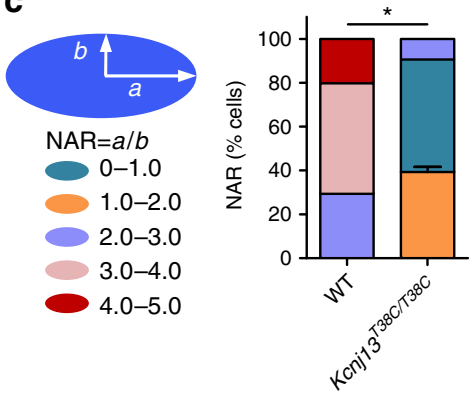

$\mathbf{g}$
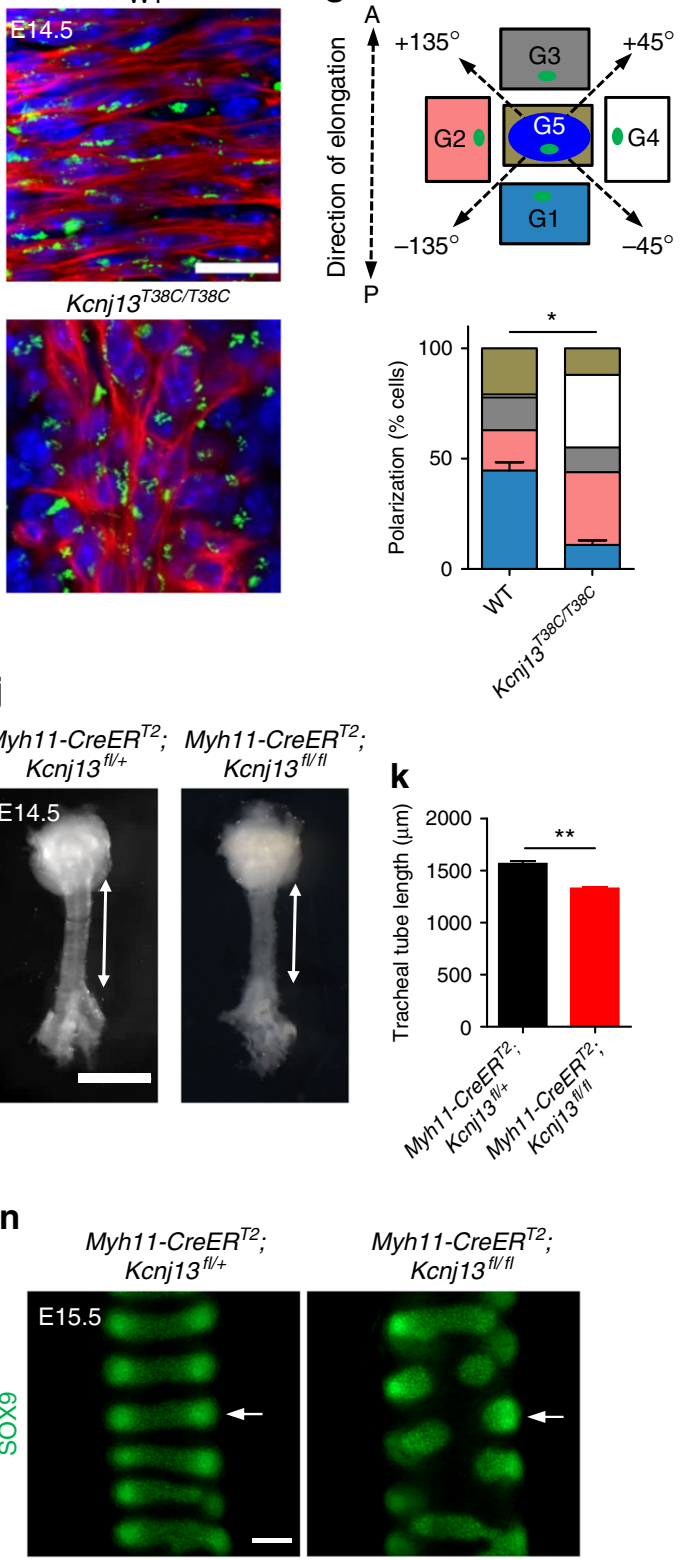

Myh11-CreER ${ }^{T 2}$; Kcnj13 $3^{f / f l}$

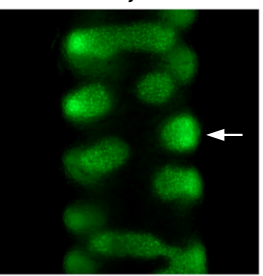


animals, indicating that mesenchymal cell condensation is not required for tracheal tube elongation.

To test whether the SM cell defects are responsible for the tracheal tube elongation phenotype in $\mathrm{Kcnj} 13^{\mathrm{T} 38 \mathrm{C} / \mathrm{T} 38 \mathrm{C}}$ mice, we used the SM-specific Cre mouse line Myh11-CreER ${ }^{T 2}$, which induces efficient recombination in airway $\mathrm{SM}$ cells ${ }^{21}$. We conditionally deleted Kcnj13 in SM by intraperitoneal tamoxifen injection for three consecutive days ( $2 \mathrm{mg}$ per day) from E11.5 to E13.5 (Fig. 4h). Mice with SM-specific Kcnj13 deletion (Fig. 4i) exhibited shorter tracheas (Fig. 4j, k), altered SM cell alignment (Fig. 4l, m) and cartilage formation defects (Fig. 4n) compared to controls, phenotypes that were not observed when Kcnj13 was deleted in epithelial cells $\left(\mathrm{Nk} \times 2.1^{\mathrm{Cre}} ; \mathrm{Kcnj13} 3^{\text {flox/flox }}\right.$ ) (Supplementary Fig. 8g-o). Moreover, Kcnj13 ${ }^{\text {T38C/T38C }}$ mice exhibited no significant differences in mitotic spindle orientation (Supplementary Fig. 9a, b) or cell proliferation in the tracheal epithelium (Supplementary Fig. 9c, d), or key growth factor gene expression (Supplementary Fig. 9e and Supplementary Table 4), indicating that Kcnj13 mutations do not affect cell growth in the epithelium. Altogether, these data indicate that KCNJ13 function is specifically required in SM, but not in epithelial cells, for tracheal tube elongation.

Both the trachea and esophagus are derived from the foregut and separate after E9.5 22,23 . Next, we sought to determine whether Kcnj13 was also required for esophageal elongation. We measured P0 esophagi and found that Kcnj13 ${ }^{T 38 C / T 38 C}$ mice displayed shortened esophageal tubes compared to their WT siblings (Supplementary Fig. 10a, b). We also analyzed the expression pattern of KCNJ13 in the developing esophagus: KCNJ13 was weakly expressed in E12.5 esophageal SM cells and this expression level increased as the SM tissue developed (Supplementary Fig. 10c). Next, we examined esophageal SM morphology. Newly differentiated esophageal SM cells were not fully elongated or well organized at E11.5-E12.5 (Supplementary Fig. 10d, e). They developed spindle shapes and became circumferentially aligned by E13.5 (Supplementary Fig. 10d, e). We found that esophageal SM was disorganized in

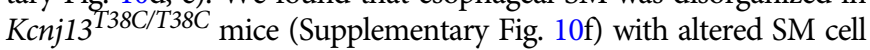
alignment (Supplementary Fig. 10g, h) and polarity (Supplementary Fig. 10i, j). These data indicate that KCNJ13-mediated SM cell alignment and polarity have a broader role in epithelial tubulogenesis.

Kcnj13 modulates actin organization in SM cells. Inactivation of Kir channels has been reported to cause depolarization of cell membranes ${ }^{24}$. To determine the molecular mechanisms underlying KCNJ13-regulated SM cell alignment and polarity, we examined SM cell membrane potential by using the voltagesensitive fluorescent dye $\mathrm{DiBAC}_{4}(3)^{25}$. The fluorescence intensity appeared higher in Kcnj13 ${ }^{\text {T38C/T38C }}$ SM cells compared to WT (Fig. 5a, b), suggesting that mutant SM cell membranes are depolarized. In addition, after treatment with $50 \mu \mathrm{M}$ VU590, a KCNJ13 inhibitor ${ }^{26}$, E14.5 WT tracheas also exhibited SM cell membrane depolarization compared to controls (Fig. 5c, d).

Membrane depolarization has been reported to decrease the amount of actin filaments (F-actin) ${ }^{27}$ and thus affect mechanical support and cell shape ${ }^{28}$. Thus, we hypothesized that SM cell membrane depolarization might lead to actin depolymerization, and consequently to alteration of cell shape and alignment. We examined F-actin content by phalloidin staining, and observed that $K c n j 13^{T 38 C / T 38 C}$ tracheas exhibited decreased F-actin levels in SM cells compared to WT (Fig. 5e, f). In addition, an ex vivo $48 \mathrm{~h}$ VU590 treatment led to a shortened trachea (Fig. 5g, h) with altered SM cell alignment (Fig. 5i, j) and shape (Fig. 5i, k), as well as decreased F-actin levels (Fig. 5i, 1), similar to the phenotypes observed in Kcnj13 $3^{T 38 C / T 38 C}$ tracheas. These data indicate that KCNJ13-regulated membrane potential modulates actin organization in tracheal SM cells.

KCNJ13, reported to have unique pore properties ${ }^{29}$, has been shown to facilitate the efflux of intracellular potassium ${ }^{30,31}$. Thus, we hypothesized that accumulation of intracellular positive charges was responsible for the altered SM cell alignment and shape phenotypes in Kcnj13 $338 C / T 38 C$ tracheas. To test this hypothesis, we used valinomycin, a potassium ionophore reported to reduce intracellular potassium levels ${ }^{32}$, to deplete intracellular potassium in Kcnj13 ${ }^{\text {T38C/T38C }}$ tracheas in an ex vivo tracheal-lung organ culture system ${ }^{33}$. After $2 \mu \mathrm{M}$ valinomycin treatment, Kcnj13 $3^{\mathrm{T} 38 \mathrm{C} / \mathrm{T} 38 \mathrm{C}}$ tracheas exhibited partially rescued SM cell alignment (Fig. $5 \mathrm{~m}, \mathrm{n}$ ) and shape (Fig. $5 \mathrm{~m}, \mathrm{o}$ ) phenotypes compared to DMSO-treated Kcnj13 ${ }^{\text {T38C/T38C }}$ tracheas. These data indicate that KCNJ13-mediated intracellular ion homeostasis is essential for tracheal SM cell alignment and shape.

Elevated extracellular potassium concentration has been reported to induce cell depolarization ${ }^{34}$ and increase intracellular potassium levels ${ }^{32}$. We hypothesized that an increase in extracellular potassium concentration might phenocopy the KCNJ13 inactivation-induced tracheal SM cell defects. We examined SM cell membrane potential in E14.5 tracheas after $40 \mathrm{mM} \mathrm{KCl}$ treatment. The intensity of fluorescence emitted from $\mathrm{DiBAC}_{4}(3)$ was higher in tracheal SM cells after $\mathrm{KCl}$ treatment compared to controls (Supplementary Fig. 11a, b). Interestingly, after a $48 \mathrm{~h} 40 \mathrm{mM} \mathrm{KCl}$ treatment, E12.5 tracheas exhibited a narrowing of SM stripes (Supplementary Fig. 11c, d), altered SM cell alignment (Supplementary Fig. 11e, f) and shape (Supplementary Fig. 11e, g) as well as decreased F-actin levels (Supplementary Fig. 11e, h) compared to controls. We then used ouabain, a well established inhibitor of $\mathrm{Na}+/ \mathrm{K}+-\mathrm{ATPase}^{32}$, to deplete intracellular potassium in the presence of elevated extracellular potassium. Notably, ouabain treatment, compared to DMSO treatment, partially rescued the SM cell alignment (Supplementary Fig. 11i, j) and shape (Supplementary Fig. 11i, k) phenotypes caused by the increase of extracellular potassium.

Fig. 4 Kcnj13 orchestrates SM cell alignment and polarity. a Immunostaining for $\alpha$ SMA (red) and DAPI staining (blue) in dorsal views of E14.5 WT ( $n=8$ ) and $K c n j 13^{T 38 C / T 38 C}(n=8)$ tracheas. b Quantification of E14.5 WT $(n=8)$ and $K c n j 13^{T 38 C / T 38 C}(n=8)$ tracheal SM cell orientation. c Quantification of E14.5 WT $(n=8)$ and Kcnj13 T38C/T38C $(n=8)$ tracheal SM cell nuclear aspect ratio (NAR). d Immunostaining for $\alpha$ SMA (red) and DAPI staining (blue) of transverse sections of E14.5 WT $(n=10)$ and $K c n j 13^{T 38 C / T 38 C}(n=10)$ tracheas. e Quantification of WT $(n=10)$ and $K c n j 13^{T 38 C} / T_{38 C}(n=10)$ tracheal SM cell layers. f Immunostaining for $\alpha \mathrm{SMA}$ (red) and GM130 (green) and DAPI staining (blue) in dorsal views of E14.5 WT $(n=8)$ and Kcnj13 T38C/T38C $(n=8)$ tracheas. $\mathbf{g}$ Quantification of WT $(n=8)$ and $K_{c n j 13}{ }^{T 38 C / T 38 C}(n=8)$ Golgi apparatus (green) position relative to the nucleus (blue). $\mathbf{h}$ Timeline for tamoxifen administration. i Immunostaining for KCNJ13 (red), $\alpha$ SMA (green), and DAPI staining (blue) of transverse sections of E14.5 control ( $n=6$ ) and Myh11-CreER ${ }^{T 2} ; K_{c n j 13}$ flox/flox $(n=6)$ tracheas. j Representative images of ventral views of E14.5 control $(n=9)$ and Myh11-CreER ${ }^{T 2} ; K_{C n j 13}$ flox $/ f l o x(n=9)$ tracheas. Double-sided arrows indicate tracheal tube length. $\mathbf{k}$ Quantification of E14.5 control $(n=9)$ and Myh11-CreER ${ }^{T 2} ; K_{\mathrm{Cnj}} 13^{\text {flox } / f l o x}(n=9)$ tracheal tube length. I Immunostaining for $\alpha \mathrm{SMA}$ (red) and DAPI staining (blue) in dorsal views of E14.5 control $(n=9)$ and Myh11-CreER ${ }^{T 2} ; K_{\mathrm{Cnj}} 13$ flox/flox $(n=9)$ tracheas. $\mathbf{m}$ Quantification of E14.5 control $(n=9)$ and Myh11-CreER ${ }^{T 2} ; K_{c n j 13}$ flox/flox $(n=9)$ tracheal SM cell orientation. $\mathbf{n}$ Immunostaining for SOX9 (green) in ventral views of E15.5 control $(n=6)$ and Myh11-CreER ${ }^{T 2} ; K_{c n j 13}$ flox/flox $(n=6)$ tracheas. Arrows point to tracheal mesenchymal condensations. Scale bars: $1000 \mu \mathrm{m}(\mathbf{j}), 100 \mu \mathrm{m}(\mathbf{n}), 50 \mu \mathrm{m}(\mathbf{d}), 20 \mu \mathrm{m}(\mathbf{a}, \mathbf{f}, \mathbf{i}, \mathbf{I}) .{ }^{\star} P<0.05 ;{ }^{\star} P<0.01$; unpaired Student's $t$-test, mean $\pm \mathrm{s} . \mathrm{d}$ 
These results further support the model that intracellular ion homeostasis is crucial for SM cell alignment and shape.

SM contraction has been reported to drive tubulogenesis ${ }^{35}$. Based on the findings that Kcnj13 is required for SM cell alignment and actin organization, we hypothesized that a disruption of SM cell orientation might lead to compromised circumferential tracheal contraction resulting in tube elongation defects in Kcnj13 ${ }^{T 38 C / T 38 C}$ mice. To examine SM contractility, we measured contractile forces in several settings. Kcnj13 $138 \mathrm{C} / \mathrm{T} 38 \mathrm{C}$ tracheas exhibited greatly reduced contractile forces compared to WT (Supplementary Fig. 12a). Notably, after $50 \mu \mathrm{M}$ VU590 treatment, WT tracheas also exhibited impaired SM contraction a

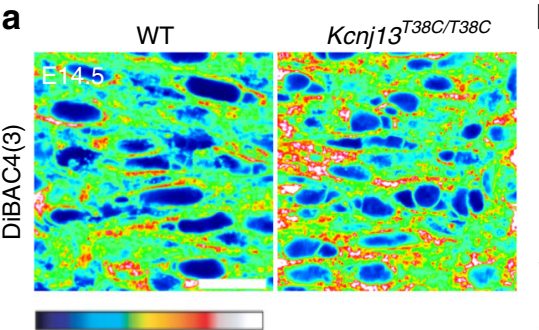

-Depolarization $\rightarrow$

e

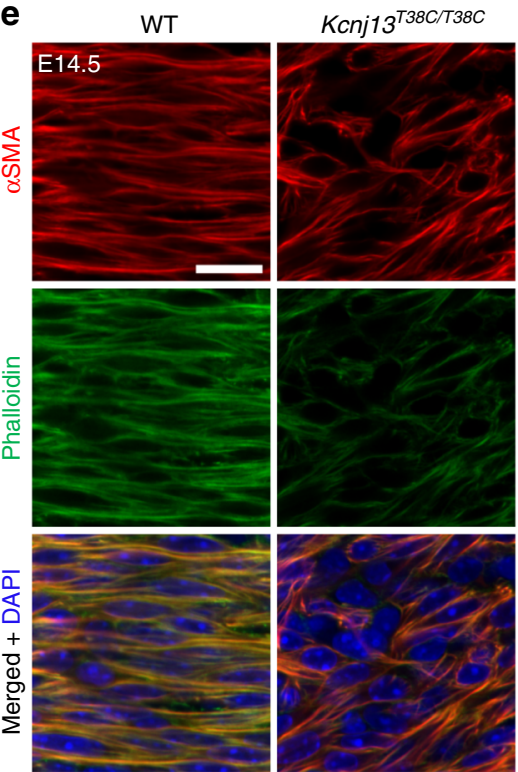

f

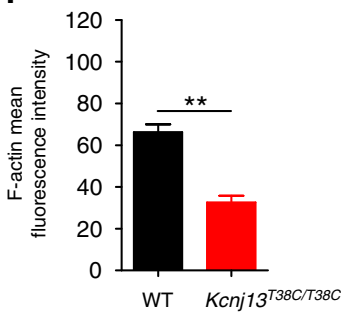

b $\quad$ c
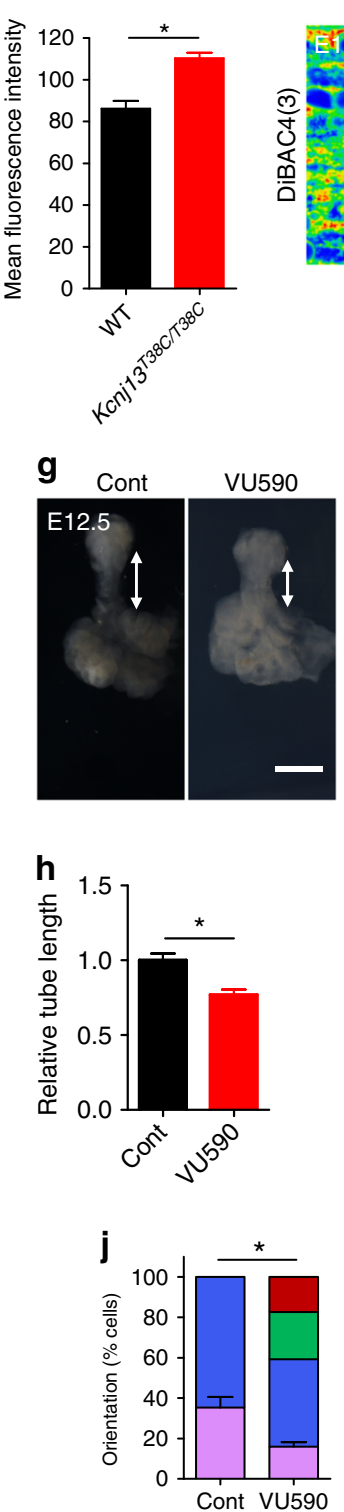

C

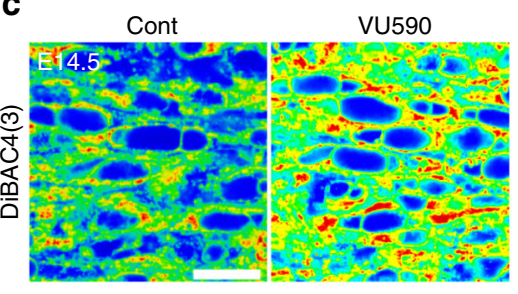

d

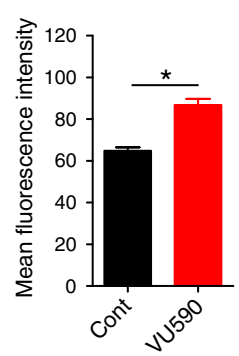

i
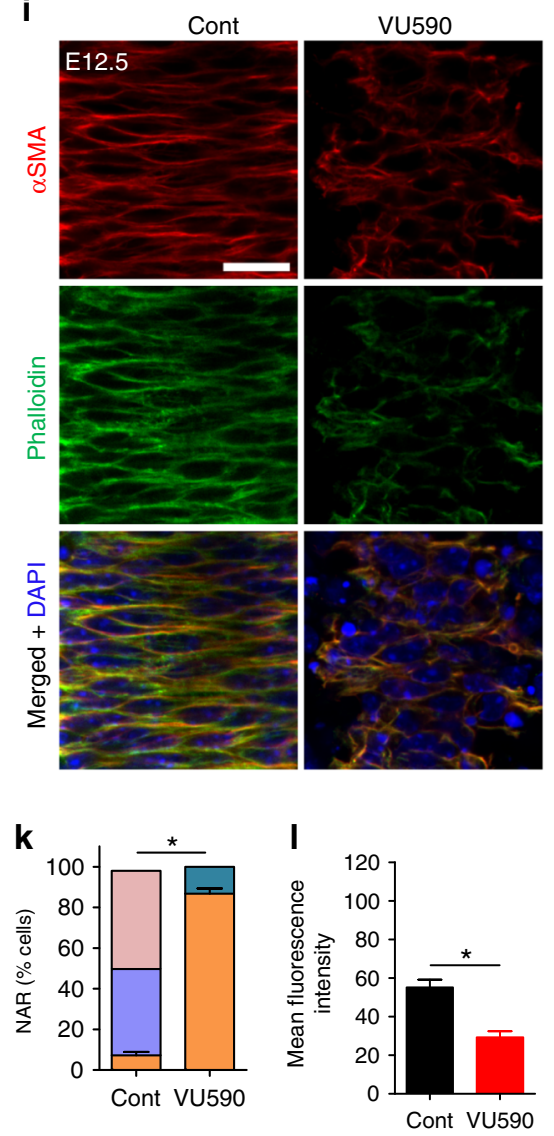
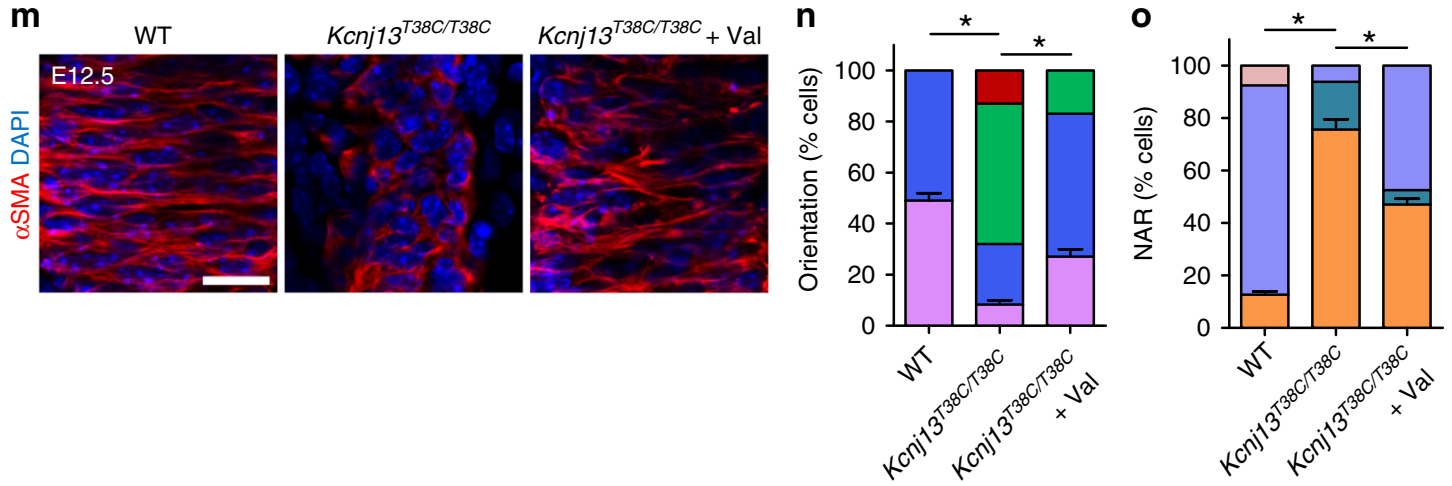
(Supplementary Fig. 12a). In addition, at early embryonic stages, the anterior part of $K c n j 13^{T 38 C / T 38 C}$ tracheas exhibited an expanded tube diameter compared to WT (Supplementary Fig. 12b), while the posterior part appeared slightly narrowed (Fig. 4d). Altogether, these data indicate that SM-mediated circumferential contraction may prevent tracheal tube overexpansion and promote its elongation along the longitudinal direction.

p-AKT as a mediator of Kcnj13 function in SM cells. We aimed to further understand how KCNJ13 could influence actin organization in tracheal SM cells. The Ser/Thr kinase AKT, an essential actin organizer ${ }^{10,11}$, has been reported to depend on potassium homeostasis for its activation state, as assessed by Ser473 phosphorylation ${ }^{32,36}$. We thus examined the phosphorylation level of AKT at Ser473 and found that it was greatly reduced in Kcnj13 $3^{T 38 C / T 38 C}$ tracheas compared to WT (Fig. 6a-d). In addition, after $50 \mu \mathrm{M}$ VU590 treatment, WT tracheas also exhibited greatly reduced phosphorylation levels of AKT at Ser473 compared to controls (Fig. 6e, f). Furthermore, Kcnj13 ${ }^{T 38 C / T 38 C}$ tracheas, after treatment with A443654, a small molecule that increases AKT phosphorylation at Ser $473^{37}$, exhibited partially rescued SM cell phenotypes (Fig. 6g-i) and Factin levels (Fig. 6j, k) compared to controls. These results indicate that reduced AKT phosphorylation in Kcnj13 $3^{\text {T38C/T38C }}$ tracheas partially accounts for the SM cell alignment and shape phenotypes via its action on actin polymerization (Fig. 6l).

\section{Discussion}

Understanding the mechanisms underlying tube formation is a fundamental goal in developmental biology. The reported roles of SM cells in tubulogenesis include wrapping nascent epithelial buds for terminal bifurcation during lung branching morphogenesis $^{38}$, and folding the epithelium into villi via mucosal buckling during the formation of the gut tube ${ }^{39}$. Kcnj13 ${ }^{\text {T38C/T38C }}$ mice exhibit a shorter trachea but no obvious defects in cell division plane or cell proliferation in the tracheal epithelium, suggesting that loss of Kcnj13 function affects some other cell type (s) during tracheal tubulogenesis. Indeed, we found that SM cellspecific inactivation of Kcnj13 phenocopied some of the defects observed in the global mutants including a shorter trachea and altered SM alignment. Mutant animals also exhibit reduced Factin levels in their tracheal SM cells as well as compromised circumferential contraction indicating major defects in these cells. However, it is important to note that the trachea of SM-specific Kcnj13 mutants is longer than that of global mutants, suggesting that Kcnj13 regulates additional cell type(s) necessary for tracheal elongation. Altogether, our data support a model whereby SM morphogenesis and contractility drive the elongation of the developing trachea; in Kcnj13 mutants, as many SM cells lose their circumferential alignment, the elongation of the tube becomes compromised, possibly as a result of both reduced circumferential contraction and increased longitudinal alignment of the SM cells, the latter of which would block elongation, as in the gut tube ${ }^{39}$. Interestingly, Wnt5a and Ror 2 mutants also exhibit a shorter trachea ${ }^{40,41}$, a phenotype recently shown to be caused by defects in SM cell morphology and polarity ${ }^{13}$. It will thus be interesting to investigate whether and how Wnt5a/Ror2 and Kcnj13 intersect in the regulation of tracheal tubulogenesis including elongation. Additional studies have shown that deletion of TMEM16A, a calcium-activated chloride channel, leads to apparently shorter tracheas as well as altered tracheal SM cell shape and organization ${ }^{42}$, while CFTR, a chloride ion channel, leads to a reduced tracheal SM cell mass ${ }^{43}$ as well as fracture of the tracheal cartilage rings ${ }^{44}$, phenotypes all observed in Kcnj13 $3^{T 38 C / T 38 C}$ tracheas. It thus appears that multiple ion channels regulate tracheal SM cells and cartilage formation.

Our data on actin organization in tracheal SM cells suggest a model by which ion channels mediate actin filament reorganization through the actin organizer AKT. Although the exact mechanisms by which KCNJ13 regulates AKT activity remain to be determined, it is possible that KCNJ13 inactivation inhibits PI3K or activates PTEN, thereby impairing AKT plasma membrane translocation and subsequent activation. Other possibilities are that KCNJ13 inactivation leads to inhibition of the mTORC2 kinase $^{45}$, or activation of the PHLPP phosphatase ${ }^{46}$.

In addition, point mutations in $K C N J 13$ are associated with several human disorders ${ }^{47-49}$. Our Kcnj13 point mutant (c. $38 \mathrm{~T}>\mathrm{C}$ (p.Leu13Pro)) exhibits severe primary tracheomalacia, providing a new model to investigate the etiology of this disease, and develop therapeutic approaches.

Overall, our data reveal the importance of potassium channels in regulating AKT phosphorylation, actin organization, and epithelial tube formation, providing further insights into the relationship between ion homeostasis and cytoskeletal organization.

\begin{abstract}
Methods
Experimental animals. All mouse husbandry was performed under standard conditions in accordance with institutional (MPG) and national ethical and animal welfare guidelines. Thirty C57BL/6J male mice treated with a $3 \times 100 \mathrm{mg} / \mathrm{kg}$ dose of $\mathrm{ENU}^{50}$ were obtained from Dr. Monica Justice (Baylor College of Medicine, Houston, TX). After a period of 10 weeks for the recovery of fertility, the mutagenized G0 males were crossed with C57BL/6J female mice. G1 males were outcrossed with C57BL/6J females to generate G2 females. Four G2 females were backcrossed with their G1 father, and the resulting G3 P0 pups were subject to

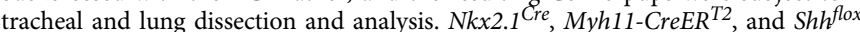
alleles have been previously described ${ }^{6,21}$. The Kcnj13 deletion (Kcnj13 ${ }^{\text {Del-E2 } \triangleleft 3}$ ) and floxed $\left(K c n j 13^{f l o x}\right)$ alleles were generated using the CRISPR-Cas9 system and homology directed repair. Two gRNAs (targets \#1 and \#2) (Supplementary Fig. 2)
\end{abstract}

Fig. 5 Kcnj13 regulates membrane potential and actin organization in SM cells. a DiBAC4(3) fluorescence in dorsal views of E14.5 WT ( $n=9$ ) and $K_{c n j 13}{ }^{T 38 C / T 38 C}(n=9)$ tracheal SM cells. b Quantification of mean DiBAC4(3) fluorescence intensity in E14.5 WT $(n=9)$ and $K c n j 13$ T38C/T38C $(n=9)$ tracheal SM cells. c DiBAC4(3) fluorescence in dorsal views of E14.5 tracheal SM cells after DMSO ( $n=6)$ or $50 \mu M$ VU590 ( $n=6)$ treatment. d Quantification of mean DiBAC4(3) fluorescence intensity in E14.5 tracheal SM cells after DMSO ( $n=6)$ or $50 \mu M$ VU590 ( $n=6)$ treatment. e Immunostaining for $\alpha \mathrm{SMA}$ (red) and phalloidin (green), and DAPI (blue) staining in dorsal views of E14.5 WT ( $n=8)$ and $K c n j 13$ T38C/T38C ( $n=8$ ) tracheas. f Quantification of mean phalloidin fluorescence intensity in E14.5 WT $(n=8)$ and $K \mathrm{cnj} 13^{T 38 \mathrm{C} / T 38 \mathrm{C}}(n=8)$ tracheal SM cells. $\mathbf{g}$ Representative images of ventral views of E12.5 tracheas after a $48 \mathrm{~h}$ DMSO $(n=6)$ or $50 \mu \mathrm{M}$ VU590 $(n=6)$ treatment. Double-sided arrows indicate tracheal tube length. h Quantification of relative tracheal tube length after a $48 \mathrm{~h}$ DMSO $(n=6)$ or $50 \mu \mathrm{M}$ VU590 ( $n=6$ ) treatment. i Immunostaining for $\alpha \mathrm{SMA}$ (red) and phalloidin (green), and DAPI (blue) staining in dorsal views of E12.5 tracheas after $48 \mathrm{~h}$ DMSO ( $n=9$ ) or $50 \mu \mathrm{M}$ VU590 ( $n=9$ ) treatment. Quantification of E12.5 tracheal SM cell orientation (j), NAR (k), and mean phalloidin fluorescence intensity $(\mathbf{I})$ after a $48 \mathrm{~h}$ DMSO $(n=9)$ or $50 \mu M$ VU590 ( $n=9$ ) treatment. $\mathbf{m}$ Immunostaining for $\alpha$ SMA (red) and DAPI staining (blue) in dorsal views of E12.5 WT ( $n=24)$ and Kcnj13 $38 \mathrm{C} /$ T38C $(n=12)$ tracheas after a $48 \mathrm{~h}$ DMSO treatment, and $K c n j 13^{T 38 C} / T 38 \mathrm{C}$ tracheas $(n=12)$ after a $48 \mathrm{~h} 2 \mu \mathrm{M}$ ionophore valinomycin treatment. Quantification of SM cell orientation (n) and NAR (o) of E12.5 WT $(n=12)$ and Kcnj13 ${ }^{T 38 C / T 38 C}(n=12)$ tracheas after a $48 \mathrm{~h}$ DMSO treatment, and Kcnj13 ${ }^{T 38} / T_{338}$ tracheas $(n=$ 12) after a $48 \mathrm{~h} 2 \mu \mathrm{M}$ ionophore valinomycin treatment. Scale bars: $500 \mu \mathrm{m}(\mathbf{e}), 20 \mu \mathrm{m}(\mathbf{a}, \mathbf{c}, \mathbf{g}, \mathbf{i}, \mathbf{m})$. ${ }^{\star} P<0.05 ; \star \star P<0.01 ;$ unpaired Student's $t$-test, mean \pm s.d. Cont: control, Val: valinomycin 
were selected for mouse Kcnj13 to direct Cas9 cleavage and insertion of loxP sites flanking exons 2 and 3 using an online CRISPR design tool (http://crispr.mit.edu/). The gRNA sequences were cloned into pDR274 (Addgene, Cambridge, MA) for gRNA production ${ }^{51}$. Next, gRNAs and Cas 9 mRNA were synthesized and microinjected into the cytoplasm of C57BL/6 inbred zygotes ${ }^{52}$, together with two 110 -bp single-stranded donors (\#1 and \#2). After injection, surviving zygotes were immediately transferred into oviducts of ICR albino pseudopregnant females.
Kcnj13 ${ }^{\text {Del-E2\& } 3}$ and Kcnj13 $3^{\text {flox }}$ alleles were detected in G0 mice and germlinetransmitted. For genotyping of $K c n j 13^{D e L-E 2 \triangleleft 3}$ mice, primers Fwd (5'-CATAAG AGTCAGCGCCTTCA-3') and Rev (5'-AGGCTCAGCTAACCAAGCATGA-3') were used to generate a $\sim 350$ bp PCR amplicon from the deletion allele.

For genotyping of Kcnj13 floxed mice, primers sets (loxP1-Fwd: 5'-AAAATTTT ACTTCTCTCAACTTCT-3'; LoxP1-Rev: 5'-AAACATTTTTGGTTT

TGTTTT-3' and loxP2-Fwd: 5'-CAACTTAGATTTATGCTTGAAA-3'; a

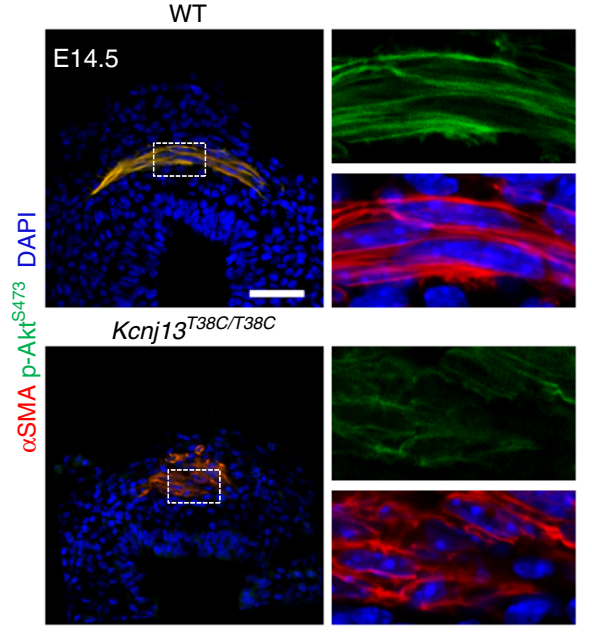

g

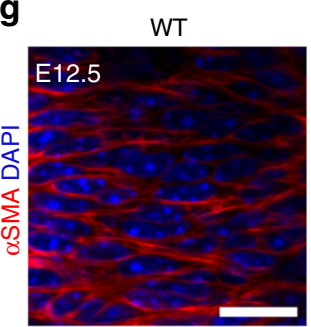

j
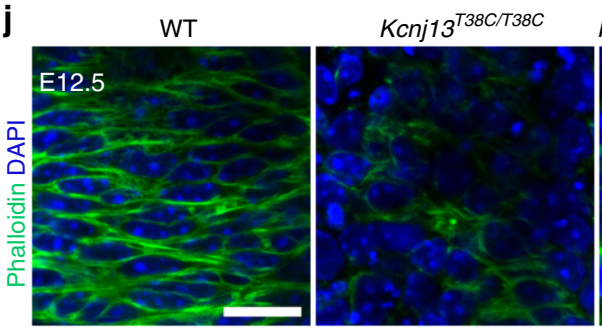

I b

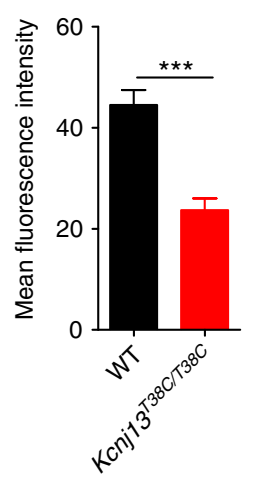

e

Akt

p-Ser473

Akt

total

GAPDH
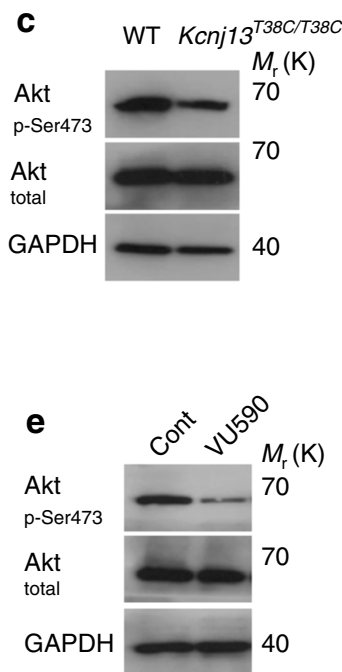

Kcnj13 $3^{\text {T38C/T38C }}+\mathrm{A} 443654$

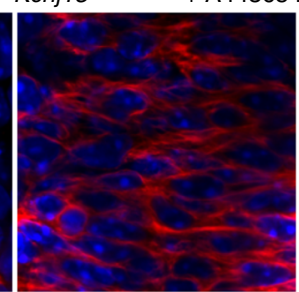

$K c n j 13^{\text {T38C/T38C }}+\mathrm{A} 443654$

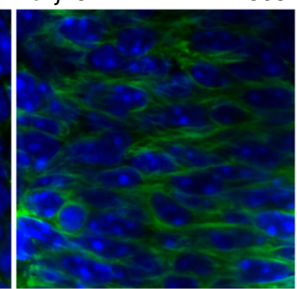

\section{h}
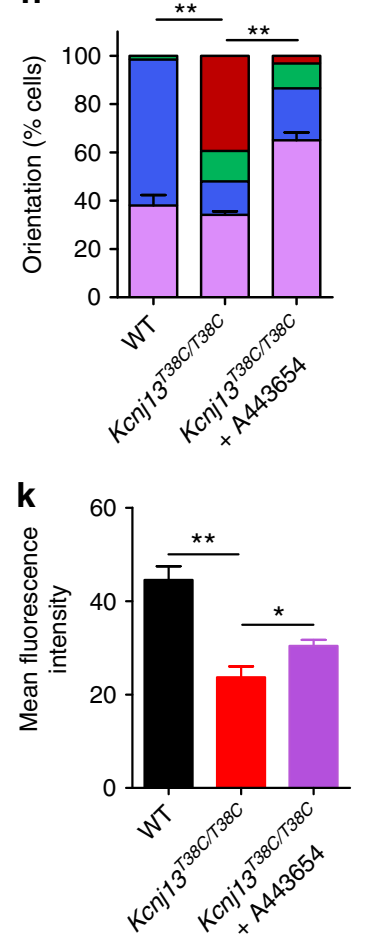

d

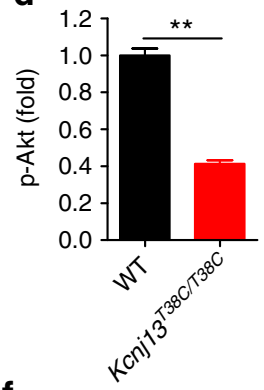

f

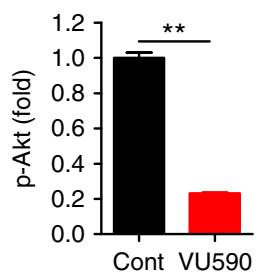

i

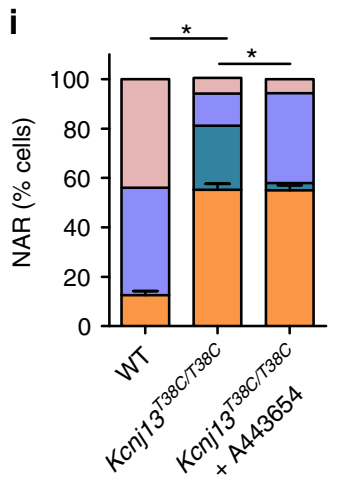

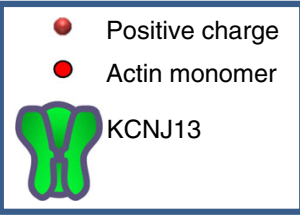
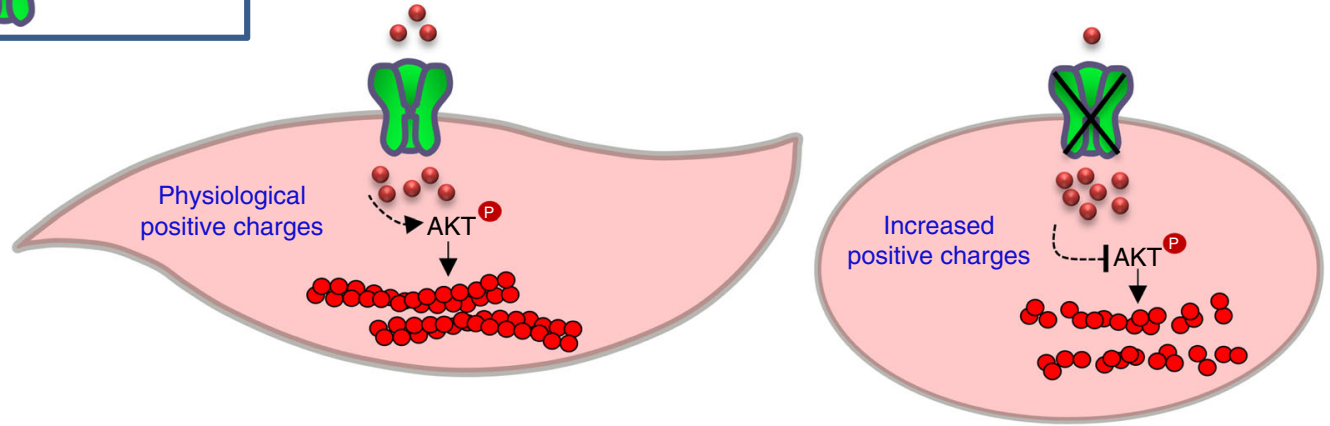
LoxP2-Rev: 5'-AAATAGACATTGATGATGTTGTT-3') were used to generate $\sim 85$ bp wild-type and $\sim 125$ bp loxP PCR amplicons for both sites. All breeding colonies were maintained under cycles of 12-hour light and 12-hour dark. All animal procedures for generating the Kcnj13 $3^{D e L-E 2 \mathcal{C}^{3}}$ and Kcnj1 $3^{f l o x}$ alleles were approved by the Institutional Animal Care and Use Committee at Baylor College of Medicine and all animal experiments were done in compliance with ethical guidelines and approved protocols.

Whole-exome sequencing analysis. Genomic DNA from two WT and two mutant mice were isolated using a standard protocol, captured using Agilent SureSelect Mouse All Exon kit V1, and sequenced using Illumina HiSeq 2000 with minimum average $50 \times$ target sequence coverage (BGI-Hong Kong). Sequence reads were aligned to the $\mathrm{C} 57 \mathrm{BL} / 6 \mathrm{~J}$ mouse reference genome $(\mathrm{mm} 10)$ and analysed using CLCBio Genomic Workbench and GATK software. To minimize false negatives, variant calls were set at $5 \times$ minimum coverage and $\geq 20 \%$ alternate reads. Sequence variants were annotated to SNPs from dbSNP (version 142) and filtered against dbSNP128.

Alcian blue staining of cartilage. Trachea cryosections $(10 \mu \mathrm{m})$ were fixed in $4 \%$ paraformaldehyde for $20 \mathrm{~min}$, treated with $3 \%$ acetic acid solution for $3 \mathrm{~min}$, stained in $0.05 \%$ alcian blue for $10 \mathrm{~min}$ and counterstained with $0.1 \%$ nuclear fast red solution for $5 \mathrm{~min}$. For whole-mount staining of tracheal cartilage, dissected tracheas were fixed in $95 \%$ ethanol for $12 \mathrm{~h}$ followed by overnight staining with $0.03 \%$ alcian blue dissolved in $80 \%$ ethanol and $20 \%$ acetic acid. Samples were cleared in $2 \% \mathrm{KOH}$

Tracheal tube length measurements. Tracheal tube length was calculated by measuring the distance between the first and last tracheal cartilage rings.

\begin{abstract}
Whole-mount immunostaining. Tracheas were isolated from E11.5 to E16.5 embryos and P0 pups and fixed in DMSO:Methanol (1:4) overnight at $4{ }^{\circ} \mathrm{C}$. Whole tracheas were then incubated in $\mathrm{H}_{2} \mathrm{O}_{2} / \mathrm{DMSO} /$ methanol (1:1:4) for $5 \mathrm{~h}$ at RT. After bleaching, samples were washed twice in $100 \%$ methanol for $1 \mathrm{~h}$ each, once in $80 \%$ methanol for $1 \mathrm{~h}$, once in $50 \%$ methanol for $1 \mathrm{~h}$, twice in PBS for $1 \mathrm{~h}$ each, and twice in $5 \% \mathrm{FBS} / \mathrm{PBS} / 0.5 \%$ Triton X-100/3\%BSA for $1 \mathrm{~h}$ each. To perform wholemount immunostaining ${ }^{53}$, tracheas were incubated with primary antibodies diluted in $5 \% \mathrm{FBS} / \mathrm{PBS} / 0.5 \%$ Triton X-100/3\% BSA for $24 \mathrm{~h}$ at $4{ }^{\circ} \mathrm{C}$, washed five times for $1 \mathrm{~h}$ each at $4^{\circ} \mathrm{C}$ with $5 \% \mathrm{FBS} / \mathrm{PBS} / 0.5 \%$ Triton X-100/3\% BSA followed by incubation with secondary antibodies for $24 \mathrm{~h}$ at $4{ }^{\circ} \mathrm{C}$, washed five times for $1 \mathrm{~h}$ each at $4{ }^{\circ} \mathrm{C}$ with $5 \% \mathrm{FBS} / \mathrm{PBS} / 0.5 \%$ Triton X-100/3\% BSA, dehydrated in methanol and then cleared in benzyl alcohol:benzyl benzoate (1:2). To visualize SM cells and tracheal mesenchymal cells, tracheas were stained for aSMA and SOX9, respectively. To visualize the Golgi apparatus, tracheas were stained for GM130. To visualize F-actin, tracheas were stained with 488 -conjugated phalloidin (Thermo Fisher Scientific, A12379)
\end{abstract}

Immunostaining of cryosections. Tracheas were dissected in PBS, fixed in $4 \%$ paraformaldehyde overnight at $4{ }^{\circ} \mathrm{C}$, incubated in $10 \%$ sucrose and $30 \%$ sucrose for $24 \mathrm{~h}$ each at $4{ }^{\circ} \mathrm{C}$, mounted in OCT embedding compound, and sectioned at $10 \mu \mathrm{m}$. To perform immunostaining, sections were fixed in $4 \%$ paraformaldehyde $10 \mathrm{~min}$ at $4{ }^{\circ} \mathrm{C}$, followed by incubation in permeabilization solution $(0.3 \%$ Triton X-100/ PBS) for $15 \mathrm{~min}$ at RT, incubated in blocking solution (5\% FBS/PBS/3\% BSA) for 1 $\mathrm{h}$ at RT, incubated in primary antibodies overnight at $4{ }^{\circ} \mathrm{C}$, washed, incubated in secondary antibodies for $2 \mathrm{~h}$ at RT, washed, and then mounted for imaging. Immunostaining for $\mathrm{p}-\mathrm{AKT} \mathrm{Ter}^{\mathrm{Se} 3}$ and aSMA was carried out with WT and mutant trachea sections on the same slide.
Airspace measurements. Six random fields per lung tissue section stained with hematoxylin and eosin from P0 WT and mutants were acquired with Axio Imager $\mathrm{Z} 2$. The percentage of terminal airspaces in the lung is the proportion of blank area of each field relative to the total area ${ }^{13}$

In situ mRNA hybridization of cryosections. Lungs were dissected in PBS, fixed in $4 \%$ paraformaldehyde overnight at $4{ }^{\circ} \mathrm{C}$, mounted in OCT embedding compound, and then sectioned at $10 \mu \mathrm{m}$. To perform in situ hybridization ${ }^{54}$, cryosections were permeabilized in $5 \mu \mathrm{g} / \mathrm{ml}$ proteinase $\mathrm{K}$ (Roche) for $15 \mathrm{~min}$ at RT, followed by acetylation for $2 \mathrm{~min}$ and pre-incubation in hybridization buffer for $3 \mathrm{~h}$ at $70^{\circ} \mathrm{C}$, incubated with DIG-labeled RNA antisense probes overnight at $70^{\circ} \mathrm{C}$, washed, incubated with alkaline phosphatase-conjugated anti-digoxigenin antibody (Roche) overnight at $4{ }^{\circ} \mathrm{C}$, washed, and then the signal was detected with NBTBCIP staining solution (Roche).

Reverse transcription quantitative PCR (RT-qPCR). Total RNA extraction was conducted using a miRNeasy Mini Kit (Qiagen). cDNA was synthesized using the Maxima First Strand cDNA Synthesis Kit (Thermo Fisher Scientific), according to manufacturer's instructions. Quantitative real-time PCR was performed using Eco Real-Time PCR System (Illumina) and Maxima SYBR Green/Fluorescein qPCR Master Mix (Thermo Fisher Scientific). The following primers were used: Actb forward 5'-CGGCCAGGTCATCACTATTGGCAAC- $3^{\prime}$ and Actb reverse 5'-GCC ACAGGATTCCATACCCAAGAAG-3'; Kcnj13 forward 5'-TGGTGAACTTTACC AGACCAGT- $3^{\prime}$ and Kcnj13 reverse 5'-GGATGTCCTCCTTTGGCAGAT-3'; Wnt5a forward 5' - CACTTAGGGGTTGTTCTCTGA-3' and Wnt5a reverse 5'ATATCAGGCACCATTAAACCA-3'; Ror2 forward 5'-CCCAACTTCTACCCAG TCCA-3' and Ror2 reverse $5^{\prime}$-TGTCCGCCACAGATGTATTG-3'; Fzd4 forward 5' - TTGTGCTATGTTGGGAACCA- $3^{\prime}$ and Fzd4 reverse 5'-GACCCCGATCTTG ACCATTA-3'; Fzd6 forward 5'-GTGCTGCAAGAGTCCTGTGA-3' and Fzd6 reverse 5'-CGCTGCTCTTTGGACTTACC-3'; Fzd8 forward 5'-ACTACAACCGC CACCGACCT-3' and $F z d 8$ reverse 5'-ACAGGCGGAGAGGAATATGA-3'; Vangll forward 5'- GATGCTGTTAGGAGGTTCGG-3' and Vangl reverse 5'-AG TCCCGCTTCTACAGCTTG-3'; Dvll forward 5'-CCTTCCATCCAAATGTTGC$3^{\prime}$ and Dvll reverse $5^{\prime}$-GTGACTGACCATAGACTCTGTGC-3'; Dvl2 forward 5'ACTGTGCGGTCTAGGTTTTGAGTC- $3^{\prime}$ and $D v / 2$ reverse $5^{\prime}$-GGAAGACGTG CCCAAGGA-3'; $D v l 3$ forward $5^{\prime}$-AGGGCCCCTGTCCAGCT- 3 ' and $D v l 3$ reverse 5'-AAAAGGCCGACTGATGGAGAT-3'; Celsrl forward 5'-ATGCTGTTGGTCA GCATGTC-3' and Celsr1 reverse 5'-GGGATCTGGACAACAACCG-3'; Celsr2 forward 5'-GCTGTGTGTGAGCATCTCGT-3' and Celsr2 reverse 5'-CATCATGA GTGTGCTGGTGT-3'; $P k 1$ forward 5'-GATGGAGAAAGCAAGCCAAG-3' and Pk1 reverse 5'-TGTGCAGCATGGAAGAGTTC-3'; Fgf10 forward 5'- CGGGAC CAAGAATGAAGACT- $3^{\prime}$ and $F g f 10$ reverse $5^{\prime}$-AGTTGCTGTTGATGGCTTTG -3'; Hgf forward 5' - AACAGGGGCTTTACGTTCACT-3' and Hgf reverse 5'- CG TCCCTTTATAGCTGCCTCC-3'; Pdgfa forward 5'-GAGGAAGCCGAGATACC CC-3' and Pdgfa reverse 5'-TGCTGTGGATCTGACTTCGAG-3'; Tgfbl forward 5'- GAGCCCGAAGCGGACTACTA-3' and Tgfbl reverse 5'-TGGTTTTCTCATA GATGGCGTTG-3'

Western blotting. Isolated P0 tracheas were lysed using RIPA buffer (Cell Signaling, 9806) supplemented with protease and phosphatase inhibitors (Cell Signaling, 5872). Lysates were centrifuged at $10,000 \times g$ for $10 \mathrm{~min}$, subjected to SDSPAGE and transferred to nitrocellulose membranes. Membranes were probed with primary and HRP-conjugated secondary antibodies (Cell Signaling Technology) and were developed using the ECL detection system (Pierce). For chemical treatment, tracheas were incubated in DMEM/F-12 medium containing $0.1 \%$ DMSO or $50 \mu \mathrm{M}$ VU590 for $4 \mathrm{~h}$ before lysis. All uncropped images related to western blotting data are available in Supplementary Fig. 13.

Fig. 6 AKT phosphorylation as a mediator of Kcnj13 function in tracheal SM cells. a Immunostaining for $\alpha$ SMA (red) and p-AKT ${ }^{\text {ser } 473}$ (green) and DAPI staining (blue) of transverse sections of E14.5 WT $(n=6)$ and Kcnj13T38C/T38C $(n=6)$ tracheas. b Quantification of mean p-AKTser473 fluorescence intensity in E14.5 WT $(n=6)$ and Kcnj13 ${ }^{\mathrm{T} 38 \mathrm{C} / \mathrm{T} 38 \mathrm{C}}(n=6)$ tracheal SM cells. c Western blotting for phosphorylated AKT, total AKT and GAPDH in PO WT $(n=12)$ and $K c n j 73^{T 38 C / T 38 C}(n=12)$ tracheas. d Quantification of relative p-AKTser473 levels in PO WT $(n=12)$ and $K c n j 13^{T 38 C / T 38 C}(n=12)$ tracheas. e Western blotting for phosphorylated AKT, total AKT and GAPDH in PO tracheas after DMSO $(n=16)$ or $50 \mu$ M VU590 ( $n=16)$ treatment. f Quantification of relative p-AKT ${ }^{\text {ser } 473}$ levels in PO tracheas after DMSO $(n=16)$ or $50 \mu M$ VU590 $(n=16)$ treatment. $\mathbf{g}$ Immunostaining for $\alpha \mathrm{SMA}$ (red) and DAPI staining (blue) in dorsal views of E12.5 WT $(n=16)$ and Kcnj13 T38C/T38C $(n=8)$ tracheas after a $48 \mathrm{~h}$ DMSO treatment, and Kcnj13 ${ }^{T 38 C / T 38 C}$ $(n=8)$ tracheas after a $48 \mathrm{~h} 0.5 \mu \mathrm{M} \mathrm{A} 443654$ treatment. Quantification of SM cell orientation $(\mathbf{h})$ and NAR $(\mathbf{i})$ of E12.5 WT $(n=8)$ and Kcnj13 $338 \mathrm{C} / \mathrm{T3} 8 \mathrm{C}$ $(n=8)$ tracheas after a $48 \mathrm{~h}$ DMSO treatment, and $K c n j 13^{T 38 C / T 38 C}(n=8)$ tracheas after a $48 \mathrm{~h} 0.5 \mu \mathrm{M} \mathrm{A} 443654$ treatment. $\mathbf{j}$ Phalloidin $(\mathrm{green})$ and DAPI staining (blue) in dorsal views of E12.5 WT $(n=8)$ and $K c n j 13^{T 38 C / T 38 C}(n=8)$ tracheal SM cells after a 48 h DMSO treatment, and Kcnj13 ${ }^{T 38 C / T 38 C}$ $(n=8)$ tracheal SM cells after a $48 \mathrm{~h} 0.5 \mu \mathrm{M}$ A443654 treatment. k Quantification of mean phalloidin fluorescence intensity in SM cells (as in j). I A working model for the KCNJ13/AKT pathway in tracheal SM cells. KCNJ13 is expressed in tracheal SM cells to maintain intracellular positive charges at physiological levels, which leads to AKT phosphorylation and actin polymerization, which are essential for SM cell alignment and polarity. When KCNJ13 is inactivated, intracellular positive charge levels increase, leading to AKT hypophosphorylation and actin depolymerization, ultimately resulting in altered SM cell alignment and polarity during tubulogenesis. Scale bars: $50 \mu \mathrm{m}(\mathbf{a}), 20 \mu \mathrm{m}(\mathbf{g}, \mathbf{j}) .{ }^{\star} P<0.05 ;{ }^{\star \star} P<0.01 ;{ }^{\star \star \star} P<0.001$; unpaired Student's $t$-test, mean \pm s.d. 
Quantification of western blot signals. The AKT, p-AKT and GAPDH levels were quantified using ImageJ. AKT and p-AKT levels were normalized to the values yielded by GAPDH. p-AKT fold-change was calculated by the ratio of $\mathrm{p}$ $\mathrm{AKT} / \mathrm{AKT}$ and WT was assigned to 1.

Antibodies. The following antibodies were used: Mouse anti-aSMA-Cy3 (1:1000, Sigma-Aldrich, C6198); Rat anti-CDH1 (1:500, Santa Cruz, sc-59778); Goat antiKCNJ13 (1:50, Santa Cruz, C19); Rabbit anti-SOX9 (1:400, Millipore, AB5535); Sheep anti-GM130 (1:50, R\&D systems, AF8199); Rabbit anti-Ki67 (1:400, Cell Signaling Technologies, \#9027); Rabbit anti Cleaved Caspase-3 (1:600, Cell Signaling Technologies, \#9661); Rabbit anti KRT5 (1:1000, Abcam, ab53121); Goat anti-CC10 (1:200, Santa Cruz, T-18); Rabbit anti-NKX2.1 (1:400, Santa Cruz, H190); Rabbit anti-SFTPC (1:400, Millipore, AB3786); Mouse anti-PCNA (1:400, Santa Cruz, sc-56); Rabbit anti-PH3 (1:400, Millipore, 06-570); Rabbit anti-pAKT $^{\text {er473 }}$ (1:200, Cell Signaling Technologies, \#4060); Rabbit anti-AKT (1:2000 Cell Signaling Technologies, \#9272), and Rabbit anti-GAPDH (1:3000, Cell Signaling Technologies, \#2118)

\section{Explant culture of mouse embryonic tracheas and lungs, and chemical} treatment. Tracheas and lungs were isolated from E12.5 embryos and cultured using an established protocol ${ }^{33}$. For KCl (P9541, Sigma) treatment, a 2 M stock solution was diluted to $40 \mathrm{mM}$. For VU590 (3891, TOCRIS) treatment, a $40 \mathrm{mM}$ stock solution was diluted to $40 \mu \mathrm{M}$. For valinomycin (3373, TOCRIS) treatment, a $2 \mathrm{mM}$ stock solution was diluted to $2 \mu \mathrm{M}$. For A443654 (16499, Cayman Chemical) treatment, a $0.5 \mathrm{mM}$ stock solution was diluted to $0.5 \mu \mathrm{M}$. Isolated tracheas and lungs were cultured in DMEM/F-12 medium containing the above chemicals at 37 ${ }^{\circ} \mathrm{C}$ in a $5 \% \mathrm{CO}_{2}$ incubator for $48 \mathrm{~h}$. For ouabain (1076, TOCRIS) treatment, a 100 $\mathrm{mM}$ stock solution was diluted to $200 \mu \mathrm{M}$ in DMEM/F-12 medium and isolated tracheas and lungs were pre-incubated for $30 \mathrm{~min}$ and then cultured for another 48 $\mathrm{h}$ after adding $40 \mathrm{mM} \mathrm{KCl}$. $0.1 \%$ DMSO in DMEM/F-12 medium was used as a control for VU590, valinomycin and A443654 treatment. 0.2\% DMSO in DMEM/ F-12 medium was used as a control for ouabain treatment. The medium was replaced every $24 \mathrm{~h}$ before collection for analysis.

Tracheal SM cell membrane potential measurements. For the examination of tracheal SM cell membrane potential, E14.5 tracheas and lungs were incubated in $10 \mu \mathrm{g} / \mathrm{ml}$ bis-(1,3-dibutylbarbituric acid) trimethine oxonol [DiBAC4(3)] (D8189, Sigma) diluted in PBS, in the dark for $30 \mathrm{~min}$ at RT. For VU590 treatment, tracheas and lungs were pre-incubated in $50 \mu \mathrm{M}$ VU590 diluted in PBS for $2.5 \mathrm{~h}$ and incubated for another $0.5 \mathrm{~h}$ after adding $10 \mu \mathrm{g} / \mathrm{ml}$ DiBAC4(3). For $\mathrm{KCl}$ treatment, tracheas and lungs were pre-incubated in $40 \mathrm{mM} \mathrm{KCl}$ diluted in PBS for $1 \mathrm{~h}$ and incubated for another $0.5 \mathrm{~h}$ after adding $10 \mu \mathrm{g} / \mathrm{ml}$ DiBAC4(3). Samples were washed twice in PBS for $10 \mathrm{~min}$ each before collection for analysis.

Imaging. Imaging of whole-mount tracheas and trachea sections was performed using a Nikon SMZ25 or Zeiss 880 upright laser scanning confocal microscope. Quantification of lumen area, tube length, SM area, SM cell orientation and nuclear aspect ratio (NAR), Golgi-apparatus position relative to the nucleus, and immunofluorescence intensity was performed using ImageJ (http://rsbweb.nih.gov/ij/).

Ex vivo trachea physiology. A total of $2 \mathrm{~mm}$ sections of tracheas were isolated from P0 pups and kept in Krebs solution $(119 \mathrm{mM} \mathrm{NaCl}, 4.7 \mathrm{mM} \mathrm{KCl}, 2.5 \mathrm{mM}$ $\mathrm{CaCl}_{2}, 1.17 \mathrm{mM} \mathrm{MgSO}_{4}, 20 \mathrm{mM} \mathrm{NaHCO} 3,1.18 \mathrm{mM} \mathrm{KH}_{2} \mathrm{PO}_{4}, 0.027 \mathrm{mM}$ EDTA, $11 \mathrm{mM}$ glucose) aerated with carbogen at $37^{\circ} \mathrm{C}$. Tracheal rings were mounted in a wire-myograph system (610-M, Danish Myo Technology) and a resting tension of $2 \mathrm{mN}$ was applied for each ring as a baseline. Contractile responses were determined by cumulative administration of indicated acetylcholine concentrations. For studies where VU590 was used, tracheas were incubated with $50 \mu \mathrm{M}$ VU590 for $10 \mathrm{~min}$ before acetylcholine exposure.

Sample size. Sample sizes for animal studies were made as large as possible based on the complex genetics. Experiments in the manuscript have $n \geq 5$ to ensure robustness. For all animal experiments, multiple animals were tested in 2-4 individual experiments.

Sample or animal exclusions. No samples or animals were excluded from the analysis.

Randomization. For all animal experiments, WT and mutant animals were chosen at random from among littermates with the appropriate genotype.

Blinding. The genotype of WT and mutant mice was unknown to the investigators before data analysis in each experiment. In the $\mathrm{KCl}$ and VU590 treatment experiments, control and $\mathrm{KCl}$ - or VU590-treated mice were littermates with the same genotype.
Statistical analysis. No statistical methods were used to predetermine sample size. Statistical analyses were performed using GraphPad software. Error bars, s.d., and $P$ values were calculated by two-tailed Student's $t$-test $\left({ }^{\star} P<0.05\right.$; ${ }^{\star *} P<0.01$; ${ }^{* *} P<$ 0.001 ; ${ }^{* * *} P<0.0001$; NS: not significant). $P<0.05$ indicates a finding is significant. $P>0.05$ indicates a finding is not significant.

Data availability. The authors declare that all data supporting the findings of this study are available within the article and its Supplementary Information files or from the corresponding authors upon reasonable request.

Received: 19 September 2017 Accepted: 25 May 2018 Published online: 19 July 2018

\section{References}

1. Brand-Saberi, B. E. \& Schäfer, T. Trachea: anatomy and physiology. Thorac. Surg. Clin. 24, 1-5 (2014).

2. Hines, E. A., Jones, M. K., Verheyden, J. M., Harvey, J. F. \& Sun, X. Establishment of smooth muscle and cartilage juxtaposition in the developing mouse upper airways. Proc. Natl Acad. Sci. USA. 110, 19444-19449 (2013).

3. Fraga, J. C., Jennings, R. W. \& Kim, P. C. Pediatric tracheomalacia. Semin. Pediatr. Surg. 25, 156-164 (2016).

4. Lubarsky, B. \& Krasnow, M. A. Tube morphogenesis: making and shaping biological tubes. Cell 112, 19-28 (2003).

5. Andrew, D. J. \& Ewald, A. J. Morphogenesis of epithelial tubes: Insights into tube formation, elongation, and elaboration. Dev. Biol. 341, 34-55 (2010).

6. Sala, F. G. et al. FGF10 controls the patterning of the tracheal cartilage rings via Shh. Development 138, 273-282 (2011).

7. Snowball, J., Ambalavanan, M., Whitsett, J. \& Sinner, D. Endodermal Wnt signaling is required for tracheal cartilage formation. Dev. Biol. 405, 56-70 (2015)

8. Dahal, G. R. et al. An inwardly rectifying $\mathrm{K}+$ channel is required for patterning. Development 139, 3653-3664 (2012).

9. Inaba, M., Yamanaka, H. \& Kondo, S. Pigment pattern formation by contactdependent depolarization. Science 335, 677 (2012).

10. Cenni, V. et al. Targeting of the Akt/PKB kinase to the actin skeleton. Cell Mol. Life Sci. 60, 2710-2720 (2003).

11. Enomoto, A. et al. Akt/PKB regulates actin organization and cell motility via Girdin/APE. Dev. Cell 9, 389-402 (2005).

12. Villanueva, S. et al. Cleft palate, moderate lung developmental retardation and early postnatal lethality in mice deficient in the Kir7.1 inwardly rectifying $\mathrm{K}+$ channel. PLoS ONE 10, e0139284 (2015).

13. Kishimoto, K. et al. Synchronized mesenchymal cell polarization and differentiation shape the formation of the murine trachea and esophagus. Nat. Commun. https://doi.org/10.1038/s41467-018-05189-2 (2018).

14. Bi, W., Deng, J. M., Zhang, Z., Behringer, R. R. \& de, Crombrugghe., B. Sox9 is required for cartilage formation. Nat. Genet. 22, 85-89 (1999).

15. Pchelintseva, E. \& Djamgoz, M. B. A. Mesenchymal stem cell differentiation control by calcium-activated potassium channels. J. Cell Physiol. 233, 37553768 (2017)

16. Nobes, C. D. \& Hall, A. Rho GTPases control polarity, protrusion, and adhesion during cell movement. J. Cell. Biol. 22, 1235-1244 (1999).

17. Chen, B., Co, C. \& Ho, C. C. Cell shape dependent regulation of nuclear morphology. Biomaterials 67, 129-136 (2015).

18. Kwon, H. B. et al. In vivo modulation of endothelial polarization by Apelin receptor signalling. Nat. Commun. 7, 11805 (2016).

19. Yang, Y. \& Mlodzik, M. Wnt-Frizzled/planar cell polarity signaling: cellular orientation by facing the wind (Wnt). Annu. Rev. Cell Dev. Biol. 31, 623-646 (2015).

20. Park, J. et al. Regulation of Sox9 by Sonic Hedgehog (Shh) is essential for patterning and formation of tracheal cartilage. Dev. Dyn. 239, 514-526 (2010).

21. Wirth, A. et al. G12-G13-LARG-mediated signaling in vascular smooth muscle is required for salt-induced hypertension. Nat. Med. 14, 64-68 (2008).

22. Que, J., Choi, M., Ziel, J. W., Klingensmith, J. \& Hogan, B. L. Morphogenesis of the trachea and esophagus: current players and new roles for noggin and Bmps. Differentiation 74, 422-437 (2006).

23. Domyan, E. T. \& Sun, X. Patterning and plasticity in development of the respiratory lineage. Dev. Dyn. 240, 477-485 (2011).

24. Hibino, H. et al. Inwardly rectifying potassium channels: their structure, function, and physiological roles. Physiol. Rev. 90, 291-366 (2010).

25. Epps, D. E., Wolfe, M. L. \& Groppi, V. Characterization of the steady-state and dynamic fluorescence properties of the potential-sensitive dye bis-(1,3dibutylbarbituric acid)trimethine oxonol (Dibac4(3)) in model systems and cells. Chem. Phys. Lipids 69, 137-150 (1994). 
26. Lewis, L. M. et al. High-throughput screening reveals a small-molecule inhibitor of the renal outer medullary potassium channel and Kir7.1. Mol. Pharmacol. 76, 1094-1103 (2009).

27. Cristofanilli, M. \& Akopian, A. Calcium channel and glutamate receptor activities regulate actin organization in salamander retinal neurons. J. Physiol. 575, 543-554 (2006).

28. Pollard, T. D. \& Cooper, J. A. Actin, a central player in cell shape and movement. Science 326, 1208-1212 (2009).

29. Krapivinsky, G. et al. A novel inward rectifier $\mathrm{K}+$ channel with unique pore properties. Neuron 20, 995-1005 (1998).

30. Shimura, M. et al. Expression and permeation properties of the $\mathrm{K}(+)$ channel Kir7.1 in the retinal pigment epithelium. J. Physiol. 531, 329-346 (2001).

31. Ghamari-Langroudi, M. et al. G-protein-independent coupling of MC4R to Kir7.1 in hypothalamic neurons. Nature 520, 94-98 (2015).

32. Eil, R. et al. Ionic immune suppression within the tumour microenvironment limits T cell effector function. Nature 537, 539-543 (2016).

33. Del Moral, P. M. \& Warburton, D. Explant culture of mouse embryonic whole lung, isolated epithelium, or mesenchyme under chemically defined conditions as a system to evaluate the molecular mechanism of branching morphogenesis and cellular differentiation. Methods Mol. Biol. 633, 71-79 (2010).

34. Poulsen, J. H. \& Williams, J. A. Effect of extracellular K+ concentration on resting potential, caerulein-induced depolarization and amylase release from mouse pancreatic acinar cells. Pflug. Arch. 370, 173-177 (1977).

35. Brozovich, F. V. et al. Mechanisms of vascular smooth muscle contraction and the basis for pharmacologic treatment of smooth muscle disorders. Pharmacol. Rev. 68, 476-532 (2016).

36. Shankar, $H$. et al. Role of $G$ protein-gated inwardly rectifying potassium channels in P2Y12 receptor-mediated platelet functional responses. Blood 104, 1335-1343 (2004).

37. Han, E. K. et al. Akt inhibitor A-443654 induces rapid Akt Ser-473 phosphorylation independent of mTORC1 inhibition. Oncogene 26, 5655-5661 (2007).

38. Kim, H. Y. et al. Localized smooth muscle differentiation is essential for epithelial bifurcation during branching morphogenesis of the mammalian lung. Dev. Cell 34, 719-726 (2015).

39. Shyer, A. E. et al. Villification: how the gut gets its villi. Science 342, 212-218 (2013).

40. Li, C., Xiao, J., Hormi, K., Borok, Z. \& Minoo, P. Wnt5a participates in distal lung morphogenesis. Dev. Biol. 248, 68-81 (2002).

41. Oishi, I. et al. The receptor tyrosine kinase Ror2 is involved in non-canonical Wnt5a/JNK signalling pathway. Genes Cells 8, 645-654 (2003).

42. Rock, J. R., Futtner, C. R. \& Harfe, B. D. The transmembrane protein TMEM16A is required for normal development of the murine trachea. Dev. Biol. 321, 141-149 (2008).

43. Pan, J., Luk, C., Kent, G., Cutz, E. \& Yeger, H. Pulmonary neuroendocrine cells, airway innervation, and smooth muscle are altered in Cftr null mice. Am. J. Respir. Cell Mol. Biol. 35, 320-326 (2006).

44. Bonvin, E. et al. Congenital tracheal malformation in cystic fibrosis transmembrane conductance regulator-deficient mice. J. Physiol. 586, 3231-3243 (2008).

45. Sarbassov, D. D., Guertin, D. A., Ali, S. M. \& Sabatini, D. M. Phosphorylation and regulation of Akt/PKB by the rictor-mTOR complex. Science 307, 1098-1101 (2005).

46. Gao, T., Furnari, F. \& Newton, A. C. PHLPP: a phosphatase that directly dephosphorylates Akt, promotes apoptosis, and suppresses tumor growth Mol. Cell 18, 13-24 (2005)

47. Hejtmancik, J. F. et al. Mutations in KCNJ13 cause autosomal-dominant snowflake vitreoretinal degeneration. Am. J. Hum. Genet. 82, 174-180 (2008).

48. Sergouniotis, P. I. et al. Recessive mutations in KCNJ13, encoding an inwardly rectifying potassium channel subunit, cause leber congenital amaurosis. Am. J. Hum. Genet. 89, 183-190 (2011)

49. Pattnaik, B. R. et al. A novel KCNJ13 nonsense mutation and loss of Kir7.1 channel function causes leber congenital amaurosis (LCA16). Hum. Mutat. 36, 720-727 (2015).
50. Kile, B. T. et al. Functional genetic analysis of mouse chromosome 11. Nature 425, 81-86 (2003)

51. Hwang, W. Y. et al. Efficient genome editing in zebrafish using a CRISPR-Cas system. Nat. Biotechnol. 31, 227-229 (2013).

52. Zhong, H., Chen, Y., Li, Y., Chen, R. \& Mardon, G. CRISPR-engineered mosaicism rapidly reveals that loss of Kcnj13 function in mice mimics human disease phenotypes. Sci. Rep. 5, 8366 (2015).

53. Metzger, R. J., Klein, O. D., Martin, G. R. \& Krasnow, M. A. The branching programme of mouse lung development. Nature 453, 745-750 (2008).

54. Komatsu, Y., Kishigami, S. \& Mishina, Y. In situ hybridization methods for mouse whole mounts and tissue sections with and without additional $\beta$-galactosidase staining. Methods Mol. Biol. 1092, 1-15 (2014).

\section{Acknowledgements}

We thank Brigid Hogan, Jason Rock, Xin Sun, Pao-Tien Chuang, Ross Metzger, Cecilia Lo, Kathryn Anderson, Diana Laird, Paul Potter, Monica Justice, and Joaquim GregoBessa for discussion and advice on the lung ENU screen; Radhan Ramadass, Jenny Pestel, and Yu Hsuan Carol Yang for imaging assistance; Thomas Braun for reagents; Rubén Marín-Juez, Zacharias Kontarakis, Andrea Rossi, Jason Kuan Han Lai, Paolo Panza, Konstantinos Gkatzis, Sabine Fisher, Sharon Meaney-Gardian, and Nana Fukuda for discussion and/or assistance. Funding for this study was provided in part by the Max Planck Society to S.O. and D.Y.R.S.

\section{Author contributions}

W.Y. and D.Y.R.S. conceived the project, designed experiments, and analyzed data; W.Y., H.-T.K., F.G., L.W., B.G., C.B., V.G., S.G. and S.O. contributed to experiments and data analysis; K.K. and M.M. shared their data regarding SM cell polarity and tracheal elongation prior to publication; S.W. performed western blot and trachea physiology experiments and data analysis; H.Z., D.R. and G.M. generated and characterized Kcnj1 $3^{f l o x}$ and Kcnj13 $3^{\text {Del-E2 } r 3}$ mice, and contributed to data analysis; J.P. and M.L. performed data analysis for whole-exome sequencing; W.Y. and D.Y.R.S. wrote the manuscript. All authors commented on the manuscript.

\section{Additional information}

Supplementary Information accompanies this paper at https://doi.org/10.1038/s41467018-05043-5.

Competing interests: The authors declare no competing interests.

Reprints and permission information is available online at http://npg.nature.com/ reprintsandpermissions/

Publisher's note: Springer Nature remains neutral with regard to jurisdictional claims in published maps and institutional affiliations.

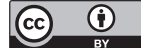

Open Access This article is licensed under a Creative Commons Attribution 4.0 International License, which permits use, sharing, adaptation, distribution and reproduction in any medium or format, as long as you give appropriate credit to the original author(s) and the source, provide a link to the Creative Commons license, and indicate if changes were made. The images or other third party material in this article are included in the article's Creative Commons license, unless indicated otherwise in a credit line to the material. If material is not included in the article's Creative Commons license and your intended use is not permitted by statutory regulation or exceeds the permitted use, you will need to obtain permission directly from the copyright holder. To view a copy of this license, visit http://creativecommons.org/ licenses/by/4.0/.

(C) The Author(s) 2018 